\title{
Structure of motion near saddle points and chaotic transport in Hamiltonian systems
}

\author{
S. S. Abdullaev \\ Institut für Plasmaphysik, Forschungszentrum Jülich GmbH, EURATOM Association, Trilateral Euregio Cluster, \\ D-52425 Jülich, Germany
}

(Received 30 March 2000)

\begin{abstract}
Generic symmetry and transport properties of near separatrix motion in $1 \frac{1}{2}$-degree-of-freedom Hamiltonian systems are studied. First the rescaling invariance of motion near saddle points, with respect to the transformation $\epsilon \rightarrow \lambda \epsilon, \chi \rightarrow \chi+\pi$ of the amplitude $\epsilon$ and phase $\chi$, of the time-periodic perturbation, is recalled. The rescaling parameter $\lambda$ depends only on the frequency of the perturbation, and the behavior of an unperturbed Hamiltonian near a saddle point. Additional rescaling symmetry of the motion with respect to transformation $\epsilon \rightarrow \lambda^{1 / 2} \epsilon, \chi \rightarrow \chi \pm \pi / 2$ is found for some Hamiltonian systems possessing symmetry in the phase space. It is shown that these rescaling invariance properties of motion lead to strong periodic (or quasiperiodic) dependencies of all statistical characteristics of the chaotic motion near the separatrix on $\log _{10} \epsilon$ with the period $\log _{10} \lambda$. These properties are examined for different models of chaotic motion by direct numerical integrations of equations of motion, as by well as using a computationally efficient method of the separatrix mapping.
\end{abstract}

PACS number(s): 05.45.Ac, 05.60.Cd, 52.25.Fi

\section{INTRODUCTION}

Most fundamental models of physics and mechanics are Hamiltonian dynamical systems. One of the essential features of deterministic dynamical systems, particularly Hamiltonian systems, is that they exhibit a chaotically unstable behavior consisting of an exponential divergency of orbits with close initial coordinates in the phase space of a system. Such a chaotic behavior of dynamical systems has been the subject of numerous studies in the 20th century, starting from pioneering works of Poincaré [1] (also see Refs. [2-4]).

A one-degree-of-freedom Hamiltonian system subjected to time-periodic perturbation (known as the $1 \frac{1}{2}$ degree-offreedom Hamiltonian system) is the most studied dynamical system. This generic system has many important applications in fluid dynamics and plasma physics. It was first realized by Aref $[5,6]$ that the Lagrangian representation of twodimensional laminar flows is equivalent to a one-dimensional Hamiltonian system, with the stream function playing the role of the Hamiltonian, and spatial coordinates as canonical variables. This analogy allowed one to apply methods of dynamical systems to study many problems in fluid dynamics and in geophysical fluid dynamics, particularly mixing of fluids [7] and transport in structured fluids containing a variety of vortices, waves, jets, and fronts [8-16].

Three-dimensional magnetic field lines in plasmas are an excellent example of the system described by the $1 \frac{1}{2}$-degree-of-freedom Hamiltonian system [17,18]. This analogy has long been used to study a chaotic destruction of magnetic surfaces in fusion devices [19], transport magnetic field lines, and heat and particles in fusion and space plasmas [20-25].

The onset of stochasticity and chaotic transport in $1 \frac{1}{2}$-degree-of-freedom Hamiltonians systems has long been the subject of numerous studies [2,26-29]. Chaotic motion appears due to the destruction of separatrices (phase-space curves connecting saddle points in the phase space of the system) by any small time-periodic perturbation, forming a zone of chaotically unstable motion near the unperturbed separatrix (the so-called stochastic layer).

One of the important features of deterministic chaotic systems is anomalous transport, i.e., a departure from the normal (Gaussian) random transport process which has been the subject of extensive studies for more than two decades [3033]. It occurs in many transport problems in fluid dynamics and physics, for example in mass transport and mixing in structured hydrodynamic flows $[10,11,13,15,16]$, transport of magnetic field lines, heat and particles in fusion and space plasmas [23-25], etc. In one dimension it is characterized by a nonlinear time dependence of a mean squared displacement $\left\langle(\Delta x)^{2}\right\rangle=2 D t^{\gamma}(\gamma \neq 1)$, while for normal diffusion $\gamma=1$, and $D$ determines a diffusion coefficient. The case $\gamma>1$ is known as enhanced (superdiffusive) transport, and the case $\gamma<1$ describes subdiffusive transport processes. It is well recognized that the anomaly of chaotic transport in deterministic Hamiltonian systems is due to the stickiness of orbits to regions of regular motion [so-called Kolmogorov-ArnoldMozer (KAM) stability islands] embedded in a stochastic layer. The structure of the chaotic domain, i.e., the mutual positions of KAM stability islands and their sizes, are believed to determine the type and rate of anomalous transport processes. The transport analysis in such complex systems has been the subject of many investigations during the last two decade (see, e.g., Refs. [32-36]).

In this work we will describe two important properties of one-degree-of-freedom Hamiltonian system subjected to time-periodic perturbation which show the fundamental connection between the structure of the phase space of system and the chaotic transport: (i) a rescaling invariance of motion near the saddle points with respect to transformations of perturbation parameters; and (ii) a quasiperiodical dependence of statistical characteristics of chaotic transport in the stochastic layer on the perturbation strength. The first property of the perturbed motion near the saddle points was recently established in Refs. [37-39]. It was found that the scaling transformation of the perturbation strength $\epsilon \rightarrow \lambda \epsilon$ and the shift of perturbation phase $\chi \rightarrow \chi+\pi$ of the time-periodic 
perturbation preserve the topology of the phase space of the canonical variables $(q, p)$ near the hyperbolic fixed point, with the rescaling law $(q, p) \rightarrow\left(\lambda^{1 / 2} q, \lambda^{1 / 2} p\right)$. The rescaling parameter $\lambda$ depends only on the frequency of perturbation and the behavior of the unperturbed system near the saddle point. We will also show that, in addition to this generic property of one-degree-of-freedom Hamiltonian systems subjected to the time-periodic perturbation, there exists an inner rescaling invariant property of motion near the saddle point with regard to transformation- $\epsilon \rightarrow \lambda^{1 / 2} \epsilon$, $\chi \rightarrow \chi \pm \pi / 2$ - which transforms the phase space of the system according to $(q, p) \rightarrow\left(\lambda^{1 / 4} q, \lambda^{1 / 4} p\right)$ if the Hamiltonian system has some sort of symmetry in the phase space $(q, p)$. These properties of perturbed motion near the saddle points mean that the topology of the stochastic layer near the saddle points, i.e., the mutual positions of KAM islands and their relatives sizes, is periodically changing with varying $\log _{10} \epsilon$ with the period $\log _{10} \lambda\left[\right.$ or $\left.\left(\log _{10} \lambda\right) / 2\right]$.

This leads to important universal consequences in a chaotic transport in the stochastic layer. Since motion slows down near the saddle points a particle spends relatively large time intervals there, thus the transport of particles along the stochastic layer is mainly determined by the structure of the stochastic layer near the saddle points. If the conjecture that similar structures of the stochastic layer gives rise to similar transport properties (for instance, the exponents $\gamma$ ), then one can expect that the statistical characteristics of chaotic transport are periodic (or quasiperiodic) functions of $\log _{10} \epsilon$ with the period determined by the rescaling parameter $\lambda$, i.e., $\log _{10} \lambda$ [or $\left.\left(\log _{10} \lambda\right) / 2\right]$. To demonstrate this property is the second aim of the present work. A short report on this subject was recently published in Ref. [40].

The work consists of seven sections. In Sec. II the universal rescaling properties of motion near the saddle points are recalled, and inner rescaling properties of motion owing to the symmetry of the system in phase space are described for the three different models containing a single saddle point, periodic saddle points in one direction of the phase space, and periodic saddle points in two directions of the phase space, respectively. These models are a particle motion in a double-well potential under external time-periodic perturbation, a three-wave model describing chaotic transport of particles in a stochastic layer formed near the separatrix of the main wave due to perturbation by two weak waves propagating in opposite direction, and the chaotic transport of passive tracers in a two-dimensional periodic vortical flow. A proof of the rescaling properties of motion near the saddle points, found by constructing the separatrix maps, is given in Sec. III and the Appendix. Statistics of the mean residence time and Poincaré recurrences in a double-well potential are studied in Sec. IV. Chaotic transport along the stochastic layer in a three-wave model is investigated in Sec. V. We study advection, second moments of spatial displacement, their exponents, and probability density functions. In Sec. VI chaotic transport in two-dimensional periodic vortical flows is studied. We have calculated periodical dependencies of the second moments of spatial displacements, and diffusion coefficients on the perturbation amplitude. In particular, regimes with strong anomalous transport, which occurs due to long distance flights, are found and analyzed. Conclusions are made in Sec. VII. (a)

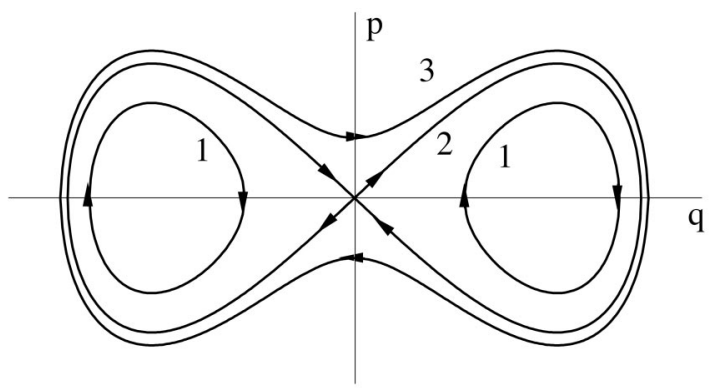

(b)

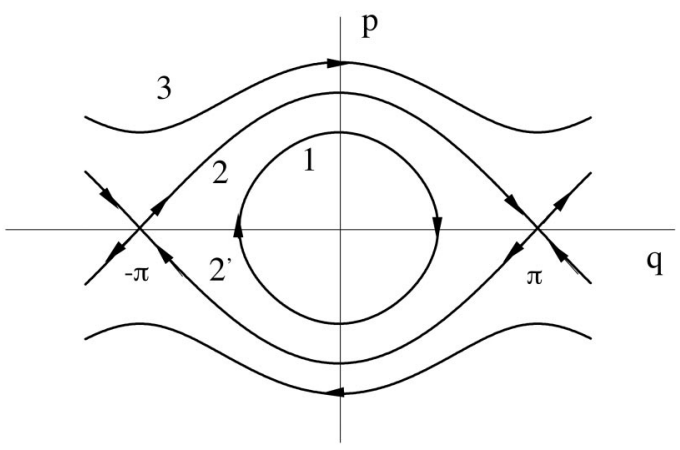

FIG. 1. Saddle connections in the phase space: (a) homoclinic orbits (curve 2), and (b) heteroclinic orbits (curves 2 and $2^{\prime}$ ) connecting different saddles points.

\section{RESCALING INVARIANCE NEAR SADDLE POINTS}

In this section we recall the rescaling invariance of motion near the saddle points, and also describe a new inner rescaling property which occurs due to the symmetry of the Hamiltonian system in the phase space. Consider a onedegree-of-freedom Hamiltonian system in the phase space of canonical variables: coordinate $q$ and momentum $p$. Suppose that a small time-periodic perturbation affects the system. The perturbed system is described by Hamiltonian equations

$$
\frac{d q}{d t}=\frac{\partial H}{\partial p}, \quad \frac{d q}{d t}=-\frac{\partial H}{\partial q}
$$

determined by the Hamiltonian function

$$
H=H_{0}(q, p)+\epsilon H_{1}\left(q, p, t+t_{0}\right),
$$

where $H_{0}(q, p)$ describes the unperturbed system, and $\epsilon H_{1}(q, p, t)=\epsilon H_{1}(q, p, t+T)$ is a time-periodic perturbation of period $T$ (or frequency $\nu=2 \pi / T$ ) with a small amplitude $\epsilon \ll 1$. In Eq. (2) we include an initial phase of perturbation $t_{0}$, the meaning of which will be clarified later.

Suppose that in the absence of perturbation $(\epsilon \equiv 0)$ there exist hyperbolic fixed points $\left(q_{s}, p_{s}\right)$ in the $(q, p)$ plane. Near each of the hyperbolic fixed points the unperturbed Hamiltonian $H_{0}(q, p)$ may be expanded in a power series of $\left(q-q_{s}\right),\left(p-p_{s}\right)$ :

$$
H_{0}(q, p)=H_{0}\left(q_{s}, p_{s}\right) \pm \frac{\alpha_{s}^{2}}{2}\left(q-q_{s}\right)^{2} \mp \frac{\beta_{s}^{2}}{2}\left(p-p_{s}\right)^{2}+O\left(\delta^{3}\right),
$$




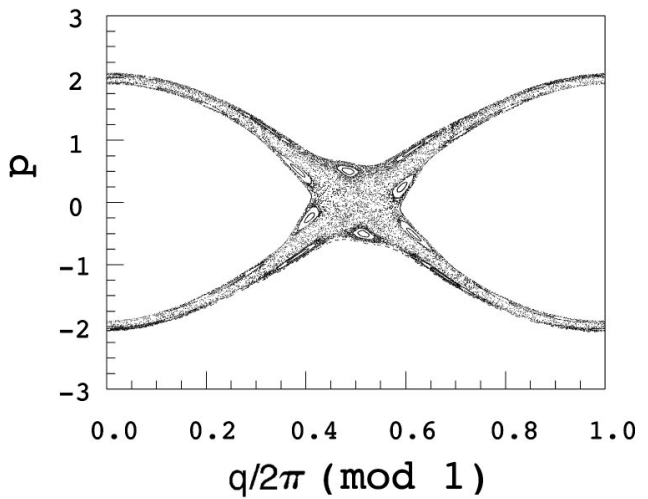

FIG. 2. Stochastic layer of system (7) for $\epsilon_{a}=0.08, \chi_{a}=-1$, and $a_{+}=a_{-}=1$.

where $\alpha_{s}$ and $\beta_{s}$ are expansion coefficients, and $O\left(\delta^{3}\right)$ is a higher order expansion term $\left[\delta \sim\left(q-q_{s}\right),\left(p-p_{s}\right)\right]$. By linear transformation,

$$
\begin{gathered}
x=\frac{1}{\sqrt{2 \alpha_{s} \beta_{s}}}\left[\alpha_{s}\left(q-q_{s}\right)+\beta_{s}\left(p-p_{s}\right)\right], \\
y=\frac{1}{\sqrt{2 \alpha_{s} \beta_{s}}}\left[-\alpha_{s} 2\left(q-q_{s}\right)+\beta_{s}\left(p-p_{s}\right)\right],
\end{gathered}
$$

Hamiltonian (3) may be also presented as

$$
H_{0}(q, p)=H_{0}\left(q_{s}, p_{s}\right) \pm \gamma_{s} x y+O\left(\delta^{3}\right),
$$
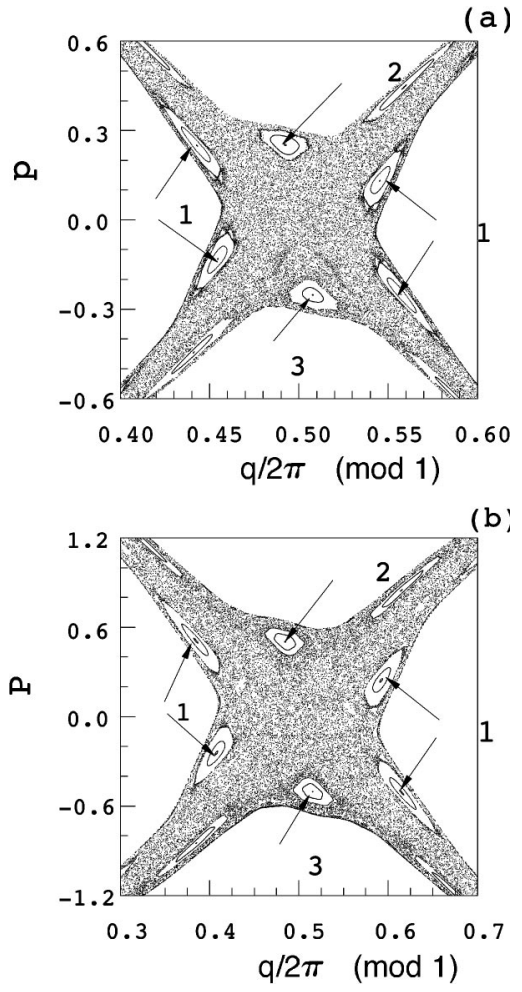

FIG. 3. Poincaré sections of the Hamiltonian system (7) near the saddle point $(q=\pi, p=0)$ : (a) $\epsilon_{a}=0.02, \chi_{a}=\pi+1$; (b) $\epsilon_{b}=\lambda \epsilon_{a}$ $=0.08, \chi_{b}=\chi_{a}-\pi$. The rescaling parameters are $\lambda=4$ and $a_{+}$ $=a_{-}=1$.

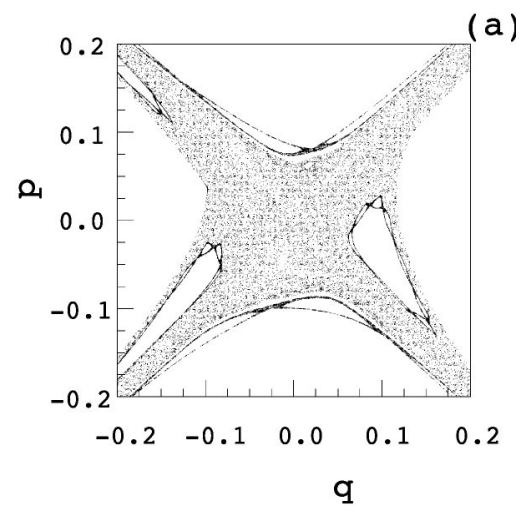

(b)

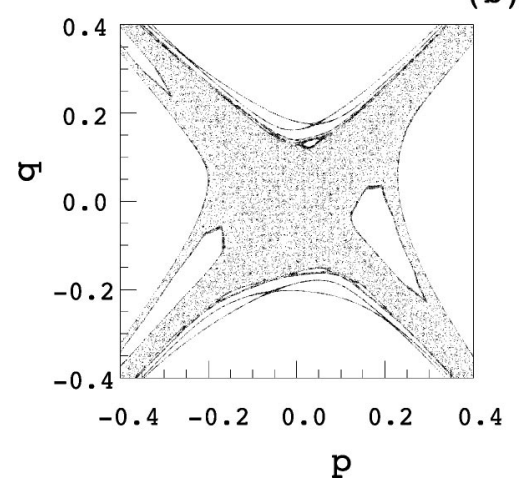

FIG. 4. Poincare sections of Hamiltonian (10) near the saddle point $(q=0, p=0)$ : (a) $\epsilon_{a}=0.0025, \chi_{a}=\pi-1$; (b) $\epsilon_{b}=\lambda^{1 / 2} \epsilon_{a}$ $=0.01, \chi_{b}=\chi_{a}-\pi / 2$. The rescaling parameter is $\lambda=16$.

where $\gamma_{s}=\alpha_{s} \beta_{s}$ is a coefficient determining the exponential growth (decrease) of coordinates near the saddle points: $x$ $\sim \exp \left( \pm \gamma_{s} t\right), y \sim \exp \left(\mp \gamma_{s} t\right)$. We further suppose that all hyperbolic fixed points have the same increments $\gamma \equiv \gamma_{s}$.

Hyperbolic fixed points $\left(q_{s}, p_{s}\right)$ which lie on the same values of the energy surface $H\left(q_{s}, p_{s}\right)=$ const may be connected. Two examples of such connections are shown in Fig. 1. The saddle connection is called a homoclinic orbit if the saddle point is connected by itself [Fig. 1(a)], or a heteroclinic orbit if it connects the different saddle points [Fig. 1(b)]. The curves connecting saddle points known as separatrices separate the regions of the phase space with different types of motion. However these separatrices are unstable to any small perturbations. In particular, a small time-periodic perturbation destroys the separatrices, and motion near the unperturbed separatrices becomes chaotic [2-4]. However, the domain of chaotic motion (or the stochastic layer) formed in the small vicinity of the unperturbed separatrices is not uniform. There are regions inside the stochastic layer with regular motions (KAM stability islands). Examples of stochastic zones are shown in Figs. 2-4. The mutual positions of these islands and their relative sizes determine the topology of the stochastic layer. As we will show below, this plays a crucial role in chaotic transport along the stochastic layer. Particularly, the structure of the stochastic layer near the saddle points mainly determines the statistical properties of chaotic motion because particles spend relatively large times to passing regions near saddle points.

\section{A. Universal rescaling invariance}

The variation of the perturbation amplitude $\epsilon$ changes not only the width of the stochastic layer but also its structure. 
However, as established recently in Refs. [37-39], this change is not arbitrary, but has a remarkable property of periodicity: the variation of the perturbation amplitude $\epsilon$ periodically changes the topology of the phase space of perturbed motion near the saddle points. That is, the structure of the phase-space of the perturbed motion near the saddle point is invariant with respect to the rescaling transformations

$$
\begin{gathered}
\epsilon \rightarrow \epsilon^{\prime}=\lambda \epsilon, \quad \chi \rightarrow \chi^{\prime}=\chi+\pi, \\
x \rightarrow x^{\prime} \approx \lambda^{1 / 2} x, \quad y \rightarrow y^{\prime} \approx \lambda^{1 / 2} y,
\end{gathered}
$$

with the rescaling parameter $\lambda=\exp (2 \pi \gamma / \nu)$ depending only on the perturbation frequency $\nu$ and the coefficient $\gamma$ in the unperturbed Hamiltonian $H_{0}(x, y)$ near the saddle point [Eq. (5)]. Here $\chi=t_{0} \nu / 2 \pi$ is the initial phase of the perturbation.

To be more specific, as a generic model consider the motion of a particle trapped by a main wave subjected to disturbances by two weak waves propagating in opposite directions. This problem is equivalent to a periodically driven pendulum. This system is described by the Hamiltonian

$$
\begin{aligned}
H= & \frac{p^{2}}{2}-\omega_{0}^{2} \cos q-\epsilon \omega_{0}^{2}\left[a_{+} \cos (q-\nu t+\chi)\right. \\
& \left.+a_{-} \cos (q+\nu t-\chi)\right],
\end{aligned}
$$

where $\omega_{0}$ is the frequency of small oscillations, and $\epsilon$ and $\nu$ represent the amplitude and the frequency of the perturbation, respectively. The quantities $a_{+}$and $a_{-}$describe amplitudes of waves propagating in positive and negative directions of the $q$ axis. The unperturbed system $(\epsilon=0)$ has elliptic fixed points at $(q=2 \pi n, p=0)$, and hyperbolic fixed points at $\left[q_{s}=2 \pi(s+1 / 2), p_{s}=0\right](n, s=0, \pm 1, \pm 2, \ldots)$ [see Fig. 1(b)]. The separatrices (curve 2) connecting the saddle points $q_{s}$ and $p_{s}$ with $q_{s \pm 1}$ and $p_{s \pm 1}$ separates the trapped orbits $\left(-\omega_{0}^{2}<H<\omega_{0}^{2}\right.$ ) (curve 1) from the untrapped ones $\left(H>\omega_{0}^{2}\right)$ (curve 3). The period of trapped orbits $T(H)$ has a following asymptotics near the separatrix $H=\omega_{0}^{2}$ :

$$
T(H)=\frac{1}{\omega_{0}} \ln \frac{32 \omega_{0}^{2}}{\left|H-\omega_{0}^{2}\right|}+O(H) \quad \text { for } \quad H \rightarrow \omega_{0}^{2} \pm 0 .
$$

The orbits on the separatrices $\left(H=\omega_{0}^{2}\right)$ are

$$
\begin{gathered}
q_{s}^{( \pm)}(t)=4 \operatorname{arccot} \frac{\exp \left[ \pm \omega_{0}\left(t-t_{0}\right)\right]+1}{\exp \left[ \pm \omega_{0}\left(t-t_{0}\right)\right]-1}, \\
p_{s}^{( \pm)}(t)= \pm \frac{2 \omega_{0}}{\cosh \left[\omega_{0}\left(t-t_{0}\right)\right]},
\end{gathered}
$$

where the signs $( \pm)$ correspond to the upper (curve 2) and lower branches (curve $2^{\prime}$ ) of the separatrix, respectively, and $t_{0}$ is a time instant when the orbit crosses a midpoint between two sequential saddle points.

Any small perturbation $(\epsilon \neq 0)$ destroys the separatrices, and forms a stochastic layer near the unperturbed separatrix. The Poincare section, i.e., points $[q(k T), p(k T)]$ of the orbit $[q(t), p(t)]$ taken at the periodic time instants $t=k T$, is shown in Fig. 2 for perturbation amplitude $\epsilon_{b}=0.08$, phase $\chi_{b}=\pi$, and perturbation frequency $\nu=4.53236 \omega_{0}$. The nu- merical integration of Hamiltonian equations were performed using the symplectic integrator method, with simplification due to the fact that Hamiltonian (7) is separable. The amplitudes of the waves are taken to be equal to each other: $a_{+}=a_{-}=1$. The structure of the stochastic layer changes with the perturbation amplitude $\epsilon$ and the phase $\chi$. However, the phase-space topologies of the stochastic layer near the hyperbolic saddle points $\left(q_{s}, p_{s}\right)$ are similar when two different perturbation amplitudes and phases are related through $(\epsilon, \chi) \rightarrow(\lambda \epsilon, \chi+\pi)$ with the rescaling parameter $\lambda$ $=\exp \left(2 \pi \omega_{0} / \nu\right)$. This rescaling property is shown in Fig. 3 by plotting Poincaré sections near the saddle point $[q$ $=\pi(\bmod 1), p=0]$ for (a) $\epsilon_{a}=0.02, \chi_{a}=0$ and (b) $\epsilon_{b}$ $=\lambda \epsilon_{a}=0.08, \chi_{b}=\pi$ at the value $\lambda=4$. The mutual positions of islands of types 1,2 , and 3 are similar; the coordinates of their fixed points are related according to Eq. (6). Due to the stickiness of orbits to islands of type 1, particles may be trapped for a long time, while stickiness to the island 2 (or 3) may lead to long distance flight along the positive (or negative) direction of the $q$ axis.

The described rescaling invariance of motion [Eq. (6)] near the saddle points is universal for one-degree-of-freedom Hamiltonian systems subjected to a small time-periodic perturbation and possessing equal expansion coefficients $\gamma$ of the unperturbed Hamiltonian $H_{0}(x, y)$ near the saddle points. This was first observed in Ref. [37] by numerical integration of the equations of motion. The proof of this property was given in Ref. [38] by constructing a so-called shifted separatrix map, and in Ref. [39] by an analytical analysis of Hamiltonian equations.

\section{B. Rescaling property due to symmetry of Hamiltonian}

If Hamiltonian system (1) has some symmetries in the phase space of canonical variables $(q, p)$, then there exists an additional rescaling invariance of the system near saddle points with respect to the transformation of the perturbation amplitude. Consider, for example, a motion of particles in a double-well potential under external time-periodic perturbation. The system is described by Hamiltonians

$$
\begin{gathered}
H=H_{0}(q, p)+\epsilon H_{1}(q, p, t), \\
H_{0}(q, p)=\frac{p^{2}}{2}-\frac{q^{2}}{2}+\frac{q^{4}}{4}, \\
H_{1}(q, p, t)=\epsilon q \cos (\nu t+\chi) .
\end{gathered}
$$

The unperturbed system $(\epsilon \equiv 0)$ has a single hyperbolic fixed point at $(q=0, p=0)$ [see Fig. 1(a)]. For $H=H_{0}(q, p)<0$ a motion is trapped in potential wells (curves 1), and for $H$ $>0$ a motion is untrapped (curve 3 ), and the separatrix ( $H$ $=0$ ) is described by curve 2 . The unperturbed period $T(H)$ of the trapped motion is equal to

$$
\begin{gathered}
T(H)=\frac{2 \sqrt{2}}{\sqrt{1+\sqrt{1+4 H}}} K(k), \\
k=\frac{\sqrt{2}(1+4 H)^{1 / 4}}{(1+\sqrt{1+4 H})^{1 / 2}},
\end{gathered}
$$


where $K(k)$ is the complete elliptic integral of the first kind with a module $k$. Outside the potential wells $(H>0)$, the period is

$$
\begin{gathered}
T(H)=\frac{4}{(1+4 H)^{1 / 4}} K(k), \\
k=\frac{\sqrt{1+\sqrt{1+4 H}}}{\sqrt{2}(1+4 H)^{1 / 4}} .
\end{gathered}
$$

Near the separatrix $(H \rightarrow 0)$, periods (11) and (12) have the asymptotics

$$
T(H)=i \ln \frac{16}{|H|}+O(H),
$$

where $i=1$ for $H<0$ and $i=2$ for $H>0$. The trajectories on the unperturbed separatrix $(H=0)$ are

$$
\begin{gathered}
q_{s}^{( \pm)}(t)= \pm \frac{\sqrt{2}}{\cosh \left(t-t_{0}\right)}, \\
p_{s}^{( \pm)}(t)=\mp \frac{\sqrt{2} \sinh \left(t-t_{0}\right)}{\cosh ^{2}\left(t-t_{0}\right)},
\end{gathered}
$$

where $t_{0}$ is a time instant when the orbit crosses the $q$ axis at the farthest distance from the saddle point.

The unperturbed Hamiltonian is symmetric with respect to $q \rightarrow-q$ and $p \rightarrow-p$, i.e., $H_{0}(-q,-p)=H_{0}(q, p)$, and but the perturbed Hamiltonian $H_{1}(q, p, t)$ is antisymmetric, i.e., $H_{1}(-q,-p, t)=-H_{1}(q, p, t)$.

The time-periodic perturbation destroys the separatrix, and the motion near by becomes chaotic. The perturbed motion near the saddle point beside the universal rescaling property [Eq. (6)] has an additional rescaling property due to the above-mentioned symmetries of Hamiltonian (10). By direct numerical integration of the equations of motion, we have found that the system near the saddle point is invariant with respect to the following transformations:

$$
\begin{gathered}
\epsilon \rightarrow \epsilon^{\prime}=\lambda^{1 / 2} \epsilon, \quad \chi \rightarrow \chi^{\prime}=\chi \pm \pi / 2, \\
q \rightarrow q^{\prime} \approx \pm \lambda^{1 / 4} p, \quad p \rightarrow p^{\prime} \approx \pm \lambda^{1 / 4} q .
\end{gathered}
$$

Poincaré sections of system (10) near the saddle point are shown in Fig. 4 for the two values of perturbation amplitude $\epsilon$ and phase $\chi$ : (a) $\epsilon_{a}=0.0025, \chi_{a}=\pi-1$ and (b) $\epsilon_{b}$ $=\lambda^{1 / 2} \epsilon_{a}=0.01, \chi_{b}=\chi_{a}-\pi / 2$. The rescaling parameter $\lambda$ $=\exp (2 \pi / \nu)$ is chosen equal to 16. Note that in Fig. 4(b) the coordinate $q$ is along the vertical axis, and the momentum $p$ is along the horizontal axis. As one can see from Fig. 4, the $q$ and $p$ axes are rescaled according to Eq. (15).

Such a rescaling invariance near the saddle point with respect to (15) occurs only due to a specific symmetry of Hamiltonian system. Considered in Sec. II A, the three-wave model also has such a property. Indeed one can show that the motion near the saddles points described by Hamiltonian (7) is invariant with respect to the transformations (15) if the amplitudes $a_{ \pm}$of perturbation waves propagating in opposite

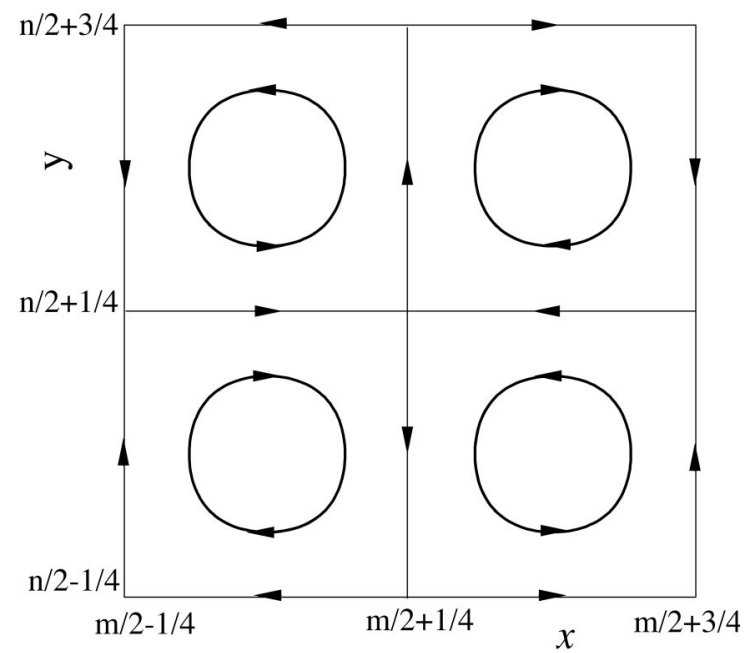

FIG. 5. Phase space of system (16).

directions are $a_{+}=-a_{-}$. The proof of the rescaling property of motion [Eq. (15)] will be given in Sec. III using the separatrix map.

\section{2D periodic vortical flow}

We study the rescaling invariance of motion described in Sec. II B in a two-dimensional (2D) periodic vortical flow. As we will see later, the existence of this property in such a flow significantly influences the chaotic transport. It is well known that the Lagrangian trajectories of fluid elements are given by the solution of the equations of motion $\dot{x}$ $=-\partial H / \partial y, \dot{y}=\partial H / \partial x$ with the streamfunction $H$ playing the role of the Hamiltonian and the spatial coordinates $(x, y)$ the roles of canonical coordinates in the Hamiltonian dynamics.

Consider a 2D periodic vortical flow subjected to a small time-periodic perturbation. The system is determined by the Hamiltonian function [9]

$$
\begin{gathered}
H=H_{0}(x, y)+\epsilon H_{1}(x, y, t), \\
H_{0}(x, y)=\frac{1}{2 \pi} \cos (2 \pi x) \cos (2 \pi y) .
\end{gathered}
$$

For convenience we have chosen the unperturbed Hamiltonian $H_{0}(x, y)$ in Eqs. (16) with the $x$ coordinate shifted by half the spatial period in comparison with one given in Refs. $[9,14]$. Hamiltonian (16) is a good model for many convective flows, including the axisymmetric Taylor vortex and the Rossby waves in geophysical fluid dynamics [41].

The phase space of the unperturbed flow is shown in Fig. 5. It has elliptic fixed points at $\left[x_{m}^{(e)}=(m-1) / 2, y_{n}^{(e)}=(n\right.$ $-1) / 2]$ and hyperbolic fixed points at $\left[x_{m}=(m-1 / 2) / 2, y_{n}\right.$ $=(n-1 / 2) / 2],(n, m=0, \pm 1, \pm 2, \ldots)$. There are four different types of saddle points: $\left(x_{m}, y_{n}\right),\left(x_{m}, y_{n+1}\right)$, $\left(x_{m+1}, y_{n}\right)$, and $\left(x_{m+1}, y_{n+1}\right)$. Because the periodicity of the system along $x$ and $y$ axes is of period 1, all other hyperbolic fixed points $\left(x_{m+2 k}, y_{n+2 p}\right),(k, p=0, \pm 1, \pm 2, \ldots)$ whose coordinates are shifted on integer numbers belong to the same classification. 

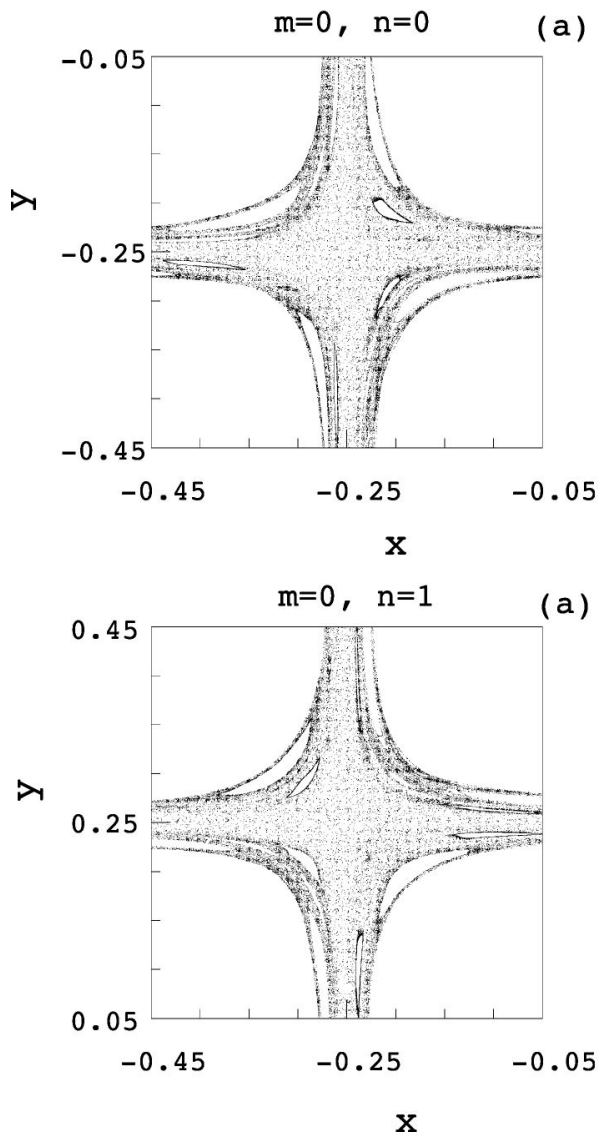

(a)

Near the saddle points the unperturbed Hamiltonian $H_{0}(x, y)$ in Eq. (16) has the following expansion in powers of $\left(x-x_{m}\right),\left(y-y_{n}\right)$ :

$$
H_{0}(x, y)=(-1)^{m+n} 2 \pi\left(x-x_{m}\right)\left(y-y_{n}\right),
$$

i.e., the parameter $\gamma$ is equal to $2 \pi$ [see Eq. (5)].

For $H=H_{0}(x, y)=0$ the saddle points are connected along horizontal $x$ and vertical $y$ axes. These saddle connections are described by orbits

$$
\cos \left[2 \pi \alpha_{s}(t)\right]= \pm 1 / \cosh \left[2 \pi\left(t-t_{0}\right)\right], \quad \alpha=x, y,
$$

where $t_{0}$ is a time instant when a trajectory passes a midpoint between two adjacent saddle points.

Inside of each cell the trajectories are closed, and have a period of motion $T(H)[\omega(H)=2 \pi / T(H)$ is a frequency]

$$
T(H)=\frac{2}{\pi} K(k), \quad k^{2}=1-4 \pi^{2} H^{2} .
$$

Near the separatrices $H \rightarrow 0$ there are the following asymptotics of $T(H)$ :

$$
T(H)=\frac{2}{\pi} \ln \frac{2}{\pi|H|}+O(H) .
$$

Any time-periodic perturbation destroys the separatrices. The motion near the unperturbed separatrices becomes chaotic, forming a stochastic web along unperturbed separatrices. The structure of the stochastic web near the saddle points is invariant with respect to the universal rescaling transformation (6) with the rescaling parameter $\lambda$ $=\exp (2 \pi \gamma / \nu)=\exp \left(4 \pi^{2} / \nu\right)$ for an arbitrary small timeperiodic perturbation $H_{1}(x, y, t)$.

For some wide class of perturbations $H_{1}(x, y, t)$, there may also exist a rescaling invariance with respect to transformations of type (15). Consider, for example, a timeperiodic perturbation of the flow [Eq. (16)] in the form of the traveling waves with the same spatial periods as the unperturbed flow and the frequency $\nu$ (a period $T=2 \pi / \nu)$,

$$
\begin{aligned}
H_{1}(x, y, t)= & \frac{\epsilon}{2 \pi}\left[a_{y} \sin (2 \pi y-\nu t-\chi)\right. \\
& \left.-a_{x} \sin (2 \pi x-\nu t-\chi)\right],
\end{aligned}
$$

where $a_{x}$ and $a_{y}$ are relative amplitudes of the travelingwave perturbations along the $x$ and $y$ axes, respectively. The perturbed Hamiltonian has the following symmetry property in $(x, y)$ space:

$$
H_{1}(x+1 / 2, y+1 / 2, t)=-H_{1}(x, y, t) .
$$

To integrate the Hamiltonian system [Eqs. (16) and (21)], we used a fifth order Bulirsch-Stoer Runge-Kutta method with an adaptive step size control, and $10^{-7}$ accuracy [42]. Poincaré sections of orbits near the saddle points are presented in Fig. 6 for two different amplitudes $\epsilon$ and phases $\chi$ of the perturbation related to the rescaling parameter $\lambda$ $=\exp \left(4 \pi^{2} / \nu\right)=16$ : (a) $\epsilon_{a}=0.0208, \chi_{a}=0$; (b) $\epsilon_{b}=\lambda^{-1 / 2} \epsilon_{a}$ $=0.0052, \chi_{b}=\chi_{a}+\pi / 2$. The relative amplitudes of waves are chosen as $a_{x}=0.5$ and $a_{y}=1$. Figure 6(a) shows Poincaré sections near the saddle points $\left(x_{m=0}, y_{n=0}\right)$ $=(-1 / 4,-1 / 4)$ and $\left(x_{m=0}, y_{n=1}\right)=(-1 / 4,1 / 4)$. Corre- 
sponding plots near the points $\left(x_{m=1}, y_{n=1}\right)=(1 / 4,1 / 4)$ and $\left(x_{m=1}, y_{n=0}\right)=(1 / 4,-1 / 4)$ are similar to those near $\left(x_{m=0}, y_{n=0}\right)$ and $\left(x_{m=0}, y_{n=1}\right)$, and they may be obtained from the latter by rotating plots by $180^{\circ}$ around the corresponding points. Figure 6(b) presents Poincaré sections near points $(1 / 4,-1 / 4)$ and $(-1 / 4,-1 / 4)$ with the inverted coordinates $(x \rightarrow-x, y \rightarrow-y)$. In the inverted coordinates $(x, y)$ they correspond to the points $\left(x_{m=0}, y_{n=1}\right)$ and $\left(x_{m=1}, y_{n=1}\right)$.

As can be seen from Fig. 6, the topology of the phase space near the saddle points is conserved with respect to the rescaling transformation:

$$
\begin{gathered}
\epsilon \rightarrow \lambda^{-1 / 2} \epsilon, \quad \chi \rightarrow \chi+\pi / 2, \\
\left(x-x_{m}\right) \rightarrow-\lambda^{-1 / 4}\left(x-x_{m^{\prime}}\right), \\
\left(y-y_{n}\right) \rightarrow-\lambda^{-1 / 4}\left(y-y_{n^{\prime}}\right) .
\end{gathered}
$$

One should note that, unlike rescaling law (15) for the system with a single saddle point, in this case the structure near the saddle point $\left(x_{m}, y_{n}\right)$ is transformed to the one near the other saddle point $\left(x_{m^{\prime}}, y_{n^{\prime}}\right)$. For the even sum $m+n$, the transformation $(m, n) \rightarrow\left(m^{\prime}, n^{\prime}\right)$ is

$$
\begin{gathered}
(m, n) \rightarrow(m, n+1), \\
(m, n+1) \rightarrow(m+1, n+1), \\
(m+1, n+1) \rightarrow(m+1, n), \\
(m+1, n) \rightarrow(m, n) .
\end{gathered}
$$

Rescaling transformations (23) and (24) occur only due to the symmetry of the perturbed Hamiltonian (22). The analysis of this property will be also given in Sec. III.

\section{SEPARATRIX MAP ANALYSIS}

For small perturbations $\epsilon$ the width of the stochastic layer is sufficiently small, and chaotic orbit are located near the unperturbed separatrices. A powerful method of analysis of such a motion is based on the separatrix mapping first introduced by Chirikov [2] using the Melnikov method [26]. The geometrical interpretation of the separatrix mapping given in Ref. [43] defines it as a return map of time $(t)$ and energy $(H)$ to the different sections of the phase space $(q, p)$. However, this conventional definition of the separatrix map does not describe the rescaling invariance of motion near saddle points, as shown in Refs. $[38,44]$. In order to overcome this shortcoming of the separatrix map, a new definition of the separatrix map, called a "shifted separatrix map," was given in Ref. [38,44]. A rigorous method of construction of Poincaré maps, particularly separatrix maps, was developed in Ref. [45]. The shifted separatrix map is defined as a map of time $(t)$ and energy $(H)$ variables to the same cross section located near saddle points. It correctly describes the rescaling invariance of motion near the saddle points. Below, based on the method developed in Ref. [45] we construct the separatrix maps for Hamiltonian systems considered in the previous sections, and using them prove the rescaling invariance of motion near saddle points. These will be also exploited in

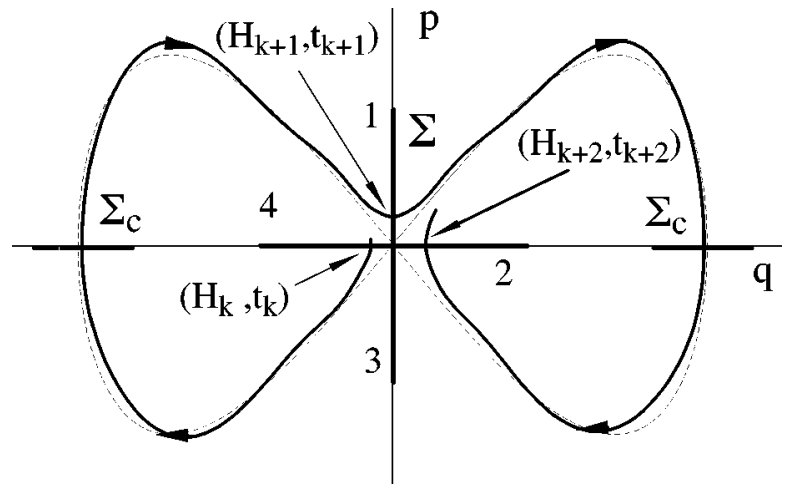

FIG. 7. Geometry of the shifted separatrix map for the doublewell potential. The solid line describes the perturbed orbit, and the dashed line corresponds to the unperturbed separatrix.

Secs. IV, V, and VI for computations of statistical characteristics of chaotic motion.

The separatrix map was used in many works to analyze the chaotic motion near the separatrix. In particular, it was applied to estimate the width of the stochastic layer $[2,46]$, and to study diffusion through a stochastic web [47] and transport in structured fluids $[14,16,10]$. Application of the shifted separatrix map to analyze the magnetic field lines in tokamaks was considered in Refs. [38,44].

The geometries of the shifted separatrix mappings are presented in Figs. 7, 9, and 10. We introduce two sections $\Sigma$ and $\Sigma_{c}$ in the $(q, p)$ plane. Section $\Sigma$ consists of two perpendicular segments of $q$ and $p$ axes, with the center at the hyperbolic fixed point $\left(q_{s}, p_{s}\right)$. Sections $\Sigma_{c}$ consist of segments perpendicular to the unperturbed separatrices $q_{s}(t)$ and $p_{s}(t)$ at the midpoint between two consecutive saddle points. Let $t_{k}$ and $H_{k}$ be a time instant and an energy at $k$ - the crossing point of the orbit with the section $\Sigma$. We define a map $\left(t_{k+1}, H_{k+1}\right)=\hat{T}_{s}\left(t_{k}, H_{k}\right)$. The map $\hat{T}_{s}$ has general forms

$$
\begin{gathered}
H_{k+1}=H_{k}+\Delta H\left(t_{k}, H_{k}\right), \\
t_{k+1}=t_{k}+\Delta t\left(H_{k}\right)+\Delta t\left(H_{k+1}\right),
\end{gathered}
$$

where $\Delta H\left(t_{k}, H_{k}\right)$ is the change of energy in the one step of the map. In the first order of the perturbation amplitude $\epsilon$, it is determined by the Melnikov integral $M(t)$ [26,4], which may be found through the generating function $G\left(t_{0}\right)$ [45], i.e.,

$$
\begin{gathered}
\Delta H\left(t_{k}, H_{k}\right)=\epsilon M\left(t_{k}+\Delta t\left(H_{k}\right)+\chi / \nu\right), \\
M\left(t_{0}\right)=\frac{\partial G\left(t_{0}\right)}{\partial t_{0}}, \\
G\left(t_{0}\right)=-\int_{-\infty}^{\infty} H_{1}\left[q_{s}(t), p_{s}(t), t\right] d t .
\end{gathered}
$$

The integral in Eqs. (26) is taken along the unperturbed separatrix $\left[q_{s}(t), p_{s}(t)\right]$ connecting the saddle points. The argument of the generating function in Eqs. (26), $t_{0}$, is a time instant when the orbit crosses the midpoint between adjacent saddle points on the unperturbed separatrix. For a timeperiodic perturbation $H_{1}(q, p, t)$ with frequency $\nu$, the func- 
tion $G(t)$ is a periodic function of $t$ with the period $2 \pi / \nu$. Here $\Delta t(H)$ is the time necessary to pass along the unperturbed orbit of energy $H$ form section $\Sigma$ to section $\Sigma_{c}$. Near the separatrix it has the universal logarithmic asymptotics

$$
\Delta t(H)=\frac{1}{2 \gamma} \ln \frac{Q}{|H|}+O(H),
$$

where the parameter $\gamma$ is the expansion coefficient of an unperturbed Hamiltonian near the saddle points [Eqs. (5)], and $Q$ is a constant depending on the system. (We suppose that at the unperturbed separatrix, $H=0)$.

Divergence of the period $\Delta t(H)$ [Eq. (27)] at $H \rightarrow 0$ is responsible for the onset of chaotic motion near the separatrix for any small perturbation amplitude $\epsilon$ due to the overlapping of infinite number of resonances of type $m \omega(H)$ $=n \nu\left[m=m_{0}, \ldots, \infty ; n\right.$ is an integer number, and $\omega(H)$ $=2 \pi / T(H)$ is the frequency of the unperturbed periodic motion] [2]. The smallest number $m_{0}$ is determined by $\epsilon$.

First we note that near the separatrix, when the logarithmic asymptotics (27) for $\Delta t(H)$ is valid, map (25) is invariant with respect to the universal rescaling transformation

$$
\epsilon \rightarrow \epsilon^{\prime}=\lambda \epsilon, \quad \chi \rightarrow \chi^{\prime}=\chi-\pi, \quad H \rightarrow H^{\prime}=\lambda H,
$$

with the rescaling parameter $\lambda=\exp (2 \pi \gamma / \nu)$. This property corresponds to the universal rescaling property of perturbed motion near the saddle point [Eq. (6)].

This means that the topology of the stochastic layer near the saddle point periodically repeats when changing the perturbation parameter $\epsilon$, i.e., it is a periodic function of $\ln \epsilon$ with the period $\ln \lambda$. It can be expected that the periodical change of the stochastic layer topology also leads to periodical (or quasiperiodical) changes in the transport characteristics. This will be studied in subsequent sections.

From Eqs. (25)-(27), it also follows that the rescaling invariance [Eqs. (28) and (6)] of the motion near the saddle point is valid when (i) the perturbation amplitude $\epsilon$ is sufficiently small that one can neglect terms of a higher order of $\epsilon$ in the change of energy $\Delta H(t, H)$, i.e., the effect in the first order of the perturbation parameter $\epsilon$; and (ii) the rescaling parameter $\lambda$ has a moderate magnitude, so that the asymptotics (27) for the rescaled energy $H^{\prime}=\lambda H$ is still valid.

One should note that the conventional definition of the separatrix map given in Ref. [2] is invariant with respect to the transformation

$$
\epsilon \rightarrow \epsilon^{\prime}=\lambda \epsilon, \quad \chi \rightarrow \chi^{\prime}=\chi, \quad H \rightarrow H^{\prime}=\lambda H,
$$

which is different from Eq. (28); therefore, it does not describe the rescaling invariance of motion near the saddle point. The main reason for this is that the time variable in the conventional separatrix map is defined on the section $\Sigma_{c}$ located far from the saddle point $[38,44]$.

\section{A. Motion in a double-well potential under external time-periodic perturbation}

First consider the construction of the separatrix map for the motion in the perturbed double-well potential (see Sec. II B). According to the geometry of the mapping shown in
Fig. 7, there exist two independent separatrix maps $T^{( \pm)}$describing the evolution of the system along separatrices on their positive, $\left[q_{s}^{(+)}(t), p_{s}^{(+)}(t)\right]$, and negative, $\left[q_{s}^{(-)}(t), p_{s}^{(-)}(t)\right]$, branches. According to Eqs. (14) and (26), the corresponding generating functions $G^{( \pm)}\left(t_{0}\right)$ are equal if the perturbation function $H_{1}(q, p, t)$ is symmetric with respect to change of signs of $q, p$, i.e., $G^{(+)}\left(t_{0}\right)$ $=G^{(-)}\left(t_{0}\right)$. In the case of antisymmetric perturbation function $H_{1}(q, p, t)$ we have $G^{(+)}\left(t_{0}\right)=-G^{(-)}\left(t_{0}\right)$.

Using Eqs. (26) and (14) for the Hamiltonian system (10), we obtain

$$
G^{( \pm)}\left(t_{0}\right)=-\int_{-\infty}^{\infty} q_{s}^{( \pm)}(t) \cos (\nu t+\chi) d t=\mp K \cos \left(\nu t_{0}+\chi\right),
$$

where

$$
K=\sqrt{2} \int_{-\infty}^{\infty} \frac{\cos (\nu \tau) d \tau}{\cosh \tau}=\frac{\pi \sqrt{2}}{\cosh (\nu \pi / 2)}
$$

The time shift $\Delta t(H)$ in Eq. (25) is equal to half the unperturbed period $T(H)$ for $H<0$ or one-fourth of $T(H)$ for $H$ $>0$; i.e., according to Eq. (13) the asymptotics of $\Delta t(H)$ near the separatrix $H \rightarrow 0$ has the form of Eq. (27), with $\gamma$ $=1$ and $Q=16$. Therefore, we have two separatrix maps $\left(H_{k+1}, t_{k+1}\right)=\hat{S}^{( \pm)}\left(H_{k}, t_{k}\right)$ describing motions near the separatrix,

$$
\begin{gathered}
H_{k+1}=H_{k} \pm \epsilon \nu K \sin \left(\varphi_{k}+\frac{\nu}{2} \ln \frac{16}{\left|H_{k}\right|}+\chi\right), \\
\varphi_{k+1}=\varphi_{k}+\frac{\nu}{2}\left[\ln \frac{16}{\left|H_{k}\right|}+\ln \frac{16}{\left|H_{k+1}\right|}\right],
\end{gathered}
$$

where the phase variable $\varphi=\nu t$ is introduced.

The separatrix maps $\hat{S}^{( \pm)}$[Eqs. (31)] introduced in addition to the universal invariance property [Eq. (28)], with the rescaling parameter $\lambda=\exp (2 \pi / \nu)$, also describe the rescaling invariance of motion [Eqs. (15)] near the saddle point due to the symmetry of the perturbed Hamiltonian $H_{1}(q, p, t)$. Below we give the proof of this property.

The rescaling transformations (15) may also be formulated as

$$
\epsilon \rightarrow \lambda^{1 / 2} \epsilon, \quad \chi \rightarrow \chi \pm \pi / 2, \quad H \rightarrow-\lambda^{1 / 2} H .
$$

We study the rescaling properties of fixed points of motion near the saddle points. Consider the cross sections of orbits at the branches $p=1,2,3$, and 4 of section $\Sigma$ shown in Fig. 7. Let $\left(\varphi_{q, s}^{(p)}, H_{q, s}^{(p)}\right)$ be a $(q, s)$ fixed point at the $p$ th branch of $\Sigma$, i.e.,

$$
\left(\varphi_{q, s}^{(p)}+2 \pi s, H_{q, s}^{(p)}\right)=\left(\hat{F}_{p}\right)^{q}\left(\varphi_{q, s}^{(p)}, H_{q, s}^{(p)}\right)
$$

where $q, s=1,2, \ldots$ The maps $\hat{F}_{p}(p=1,2,3,4)$ are formed by the consecutive application of the separatrix maps $\hat{S}^{( \pm)}$:

$$
\begin{array}{ll}
\hat{F}_{1}=\left(\hat{S}^{(-)} \hat{S}^{(+)}\right)^{q}, & \hat{F}_{2}=\left(\hat{S}^{(+)}\right)^{q}, \\
\hat{F}_{3}=\left(\hat{S}^{(+)} \hat{S}^{(-)}\right)^{q}, & \hat{F}_{4}=\left(\hat{S}^{(-)}\right)^{q} .
\end{array}
$$


(a)

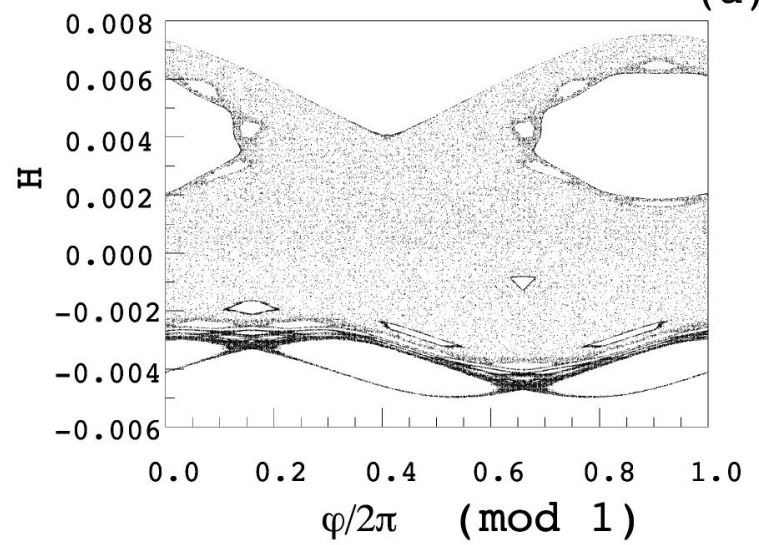

(b)

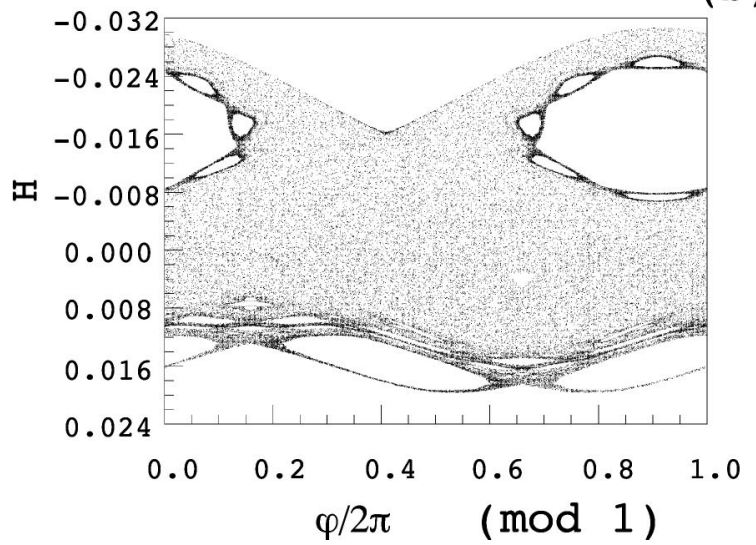

FIG. 8. Poincare sections of orbits at section $\Sigma$ obtained by the separatrix map for the same parameters as in Fig. 4.

Although each of the separatrix maps $\hat{S}^{( \pm)}$is not invariant with respect to transformation (32), their combinations of types $\hat{S}^{(\mp)} \hat{S}^{( \pm)}$and $\left(\hat{S}^{( \pm)}\right)^{2}$ are transformed as

$$
\begin{aligned}
& \left(\hat{S}^{(+)}\right)^{2} \rightarrow \hat{S}^{( \pm)} \hat{S}^{(\mp)}, \quad \hat{S}^{(-)} \hat{S}^{(+)} \rightarrow\left(\hat{S}^{(\mp)}\right)^{2}, \\
& \left(\hat{S}^{(-)}\right)^{2} \rightarrow \hat{S}^{(\mp)} \hat{S}^{( \pm)}, \quad \hat{S}^{(+)} \hat{S}^{(-)} \rightarrow\left(\hat{S}^{( \pm)}\right)^{2},
\end{aligned}
$$

which can easily be proved by direct calculations. Therefore, rescaling transformations (32) transform the maps $\hat{F}_{p}$ as

$$
\hat{F}_{1} \rightarrow \hat{F}_{4}, \quad \hat{F}_{2} \rightarrow \hat{F}_{3}, \quad \hat{F}_{3} \rightarrow \hat{F}_{2}, \quad \hat{F}_{4} \rightarrow \hat{F}_{4}
$$

for $\chi \rightarrow \chi+\pi / 2$, and

$$
\hat{F}_{1} \rightarrow \hat{F}_{2}, \quad \hat{F}_{2} \rightarrow \hat{F}_{1}, \quad \hat{F}_{3} \rightarrow \hat{F}_{4}, \quad \hat{F}_{4} \rightarrow \hat{F}_{3}
$$

for $\chi \rightarrow \chi-\pi / 2$. One can see that transformations (32), (36), and (37) are equivalent to the rescaling invariance of perturbed motion equations near the saddle point [Eqs. (15)] found by the numerical integration of equations of motion. The rescaling property [Eq. (32)] is demonstrated in Fig. 8 by plotting Poincare sections of orbits at section $\Sigma$ by the separatrix map for the same parameters as in Fig. 4: (a) $\epsilon_{a}$ $=0.025, \chi_{a}=\pi-1$; (b) $\epsilon_{b}=\lambda^{1 / 2} \epsilon_{a}=0.01, \quad \chi_{b}=\chi_{a}-\pi / 2$. Region $H>0$ corresponds to the first branch of section $\Sigma$, and $H<0$ corresponds to its fourth branch, respectively (see Fig. 7). Note that the axis $H$ in Fig. $8($ b) is inverted. One can

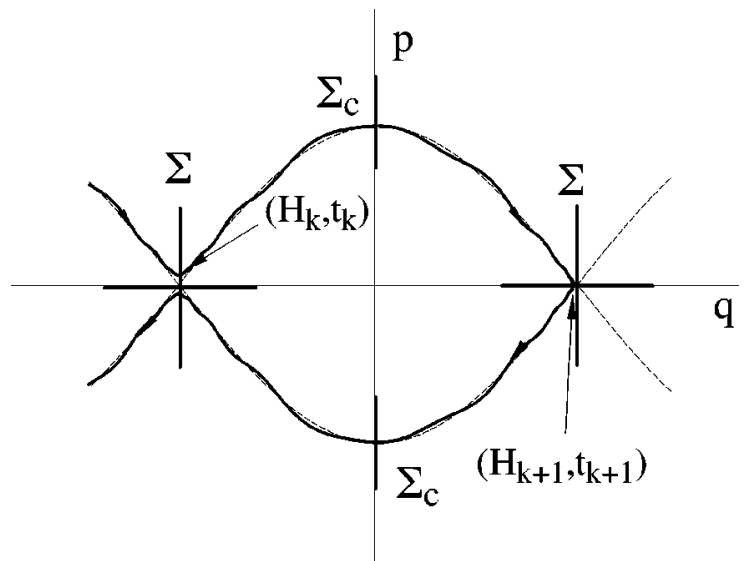

FIG. 9. Geometry of the separatrix map for a three-wave field model [Eq. (7)].

clearly see that rescaling transformations (32) indeed conserve the topology of the phase space with the rescaling law $H \rightarrow-\lambda^{1 / 2} H$.

\section{B. Separatrix map for a three-wave field model}

Here we construct a separatrix map for the particle motion in the three-wave field considered in Sec. II A. The geometry of the separatrix map is presented in Fig. 9. Calculating the generating function (26) with the Hamiltonian (7) along the unperturbed separatrices [Eqs. (9)], one can obtain

$$
\begin{gathered}
h_{k+1}=h_{k}+\epsilon K \sin \left(\varphi_{k}+\frac{\nu}{2 \omega_{0}} \ln \frac{32}{\left|h_{k}\right|}+\chi\right), \\
\varphi_{k+1}=\varphi_{k}+\frac{\nu}{2 \omega_{0}}\left[\ln \frac{32}{\left|h_{k}\right|}+\ln \frac{32}{\left|h_{k+1}\right|}\right],
\end{gathered}
$$

where $\varphi=\nu t, h=\left(H-\omega_{0}^{2}\right) / \omega_{0}^{2}$,

$$
K=\frac{4 \pi \Lambda^{2}}{\sinh (\pi \Lambda)}\left[a_{+} \exp \left(\frac{p}{|p|} \frac{\pi \Lambda}{2}\right)+a_{-} \exp \left(-\frac{p}{|p|} \frac{\pi \Lambda}{2}\right)\right]
$$

and $\Lambda=\nu / \omega_{0}$. Note that, in general, the quantity $K$ depends on the direction of the motion, i.e., on the sign of the momentum $p$.

For arbitrary values of the amplitudes $a_{ \pm}$of waves, the separatrix map is invariant with respect to the rescaling transformations (28) with the rescaling parameter $\lambda$ $=\exp \left(2 \pi \omega_{0} / \nu\right)$. However for the special case $a_{+}=-a_{-}$ noted at the end of Sec. II B, it also describes the rescaling invariance of motion [Eqs. (32)] near the saddle points due to the symmetry of the system in the phase space. Indeed, in this case we have

$$
K=\frac{p}{|p|}\left|a_{+}\right| \frac{4 \pi \Lambda^{2}}{\cosh (\pi \Lambda / 2)}
$$

and the separatrix map [Eqs. (38)] takes a form similar to Eqs. (31). For the latter we have proven the existence of the rescaling invariance [Eqs. (32)]. Application of the separatrix map (38) to study the chaotic transport in the stochastic layer will be given in Sec. V. 


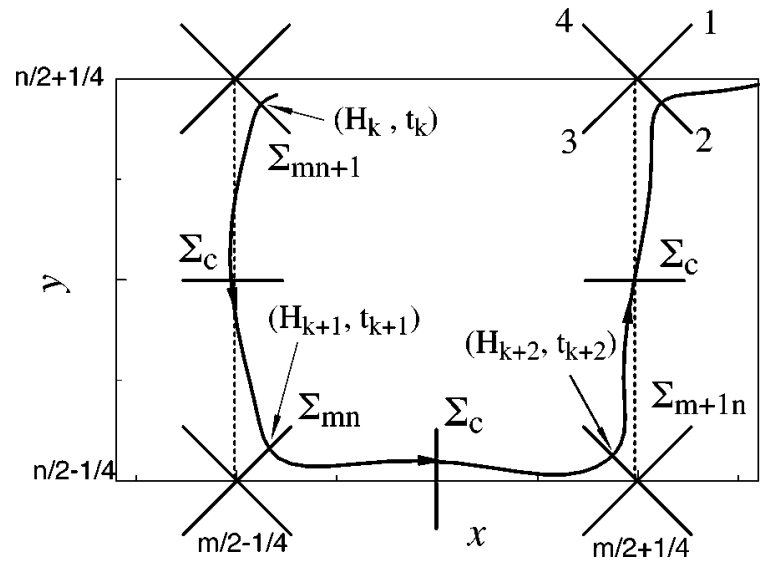

FIG. 10. Geometry of the separatrix map for the periodic vortical flow [Eq. (16)].

\section{Separatrix maps for $2 \mathrm{D}$ periodic vortical flow}

To construct the separatrix maps for this system, we introduce sections $\Sigma_{m n}(m, n=0, \pm 1, \pm 2, \ldots)$, centered at the hyperbolic fixed points $\left(x_{m}, y_{n}\right)$ shown in Fig. 10. Each of sections $\Sigma_{m n}$ consists of two segments crossing each other perpendicularly at the hyperbolic point, at $45^{\circ}$ from the $x$ axis. There are four branches of each section $\Sigma_{m n}$, denoted as $p=1,2,3$, and 4. We define maps as $Q_{k+1}^{\left(m^{\prime} n^{\prime}\right)}=\hat{S} Q_{k}^{(m n)}$, where $Q_{k}^{(m n)}$ is a crossing point of the orbit with the section $\Sigma_{m n}$. We denote them as $\hat{X}_{m, m \pm 1}^{(n)}$ and $\hat{Y}_{n, n \pm 1}^{(m)}$. The map $\hat{X}_{m, m \pm 1}^{(n)}$ transforms points $Q_{k}$ at section $\Sigma_{m n}$ to $Q_{k+1}$ at $\Sigma_{m \pm 1, n}$ along the horizontal axis $x$ at fixed $y=y_{n}$. Similarly, $\hat{Y}_{n, n \pm 1}^{(m)}$ connects points at sections $\Sigma_{m n}$ and $\Sigma_{m, n \pm 1}$ along the vertical axis $y$ at fixed $x=x_{m}$. Because of the periodicity of the system in $(x, y)$ space with period 1, we have the following symmetry properties of the maps:

$$
\begin{gathered}
\hat{X}_{m, m \pm 1}^{(n+2)}=\hat{X}_{m, m \pm 1}^{(n)}, \\
\hat{Y}_{n, n \pm 1}^{(m+2)}=\hat{Y}_{n, n \pm 1}^{(m)}, \\
\hat{X}_{m+2, m+2 \pm 1}^{(n)}=\hat{X}_{m, m \pm 1}^{(n)}, \\
\hat{Y}_{n+2, n+2 \pm 1}^{(m)}=\hat{Y}_{n, n \pm 1}^{(m)},
\end{gathered}
$$

Thanks to these symmetry properties, there exist only eight independent maps $\hat{S}$ which fully determine the dynamics of the system.

For small perturbations the map may be replaced by a separatrix map which describes the evolution of energy $(H)$ and time $(t)$ variables at sections $\Sigma_{m n}$, i.e., $\left(H_{k+1}, t_{k+1}\right)$ $=\hat{S}\left(H_{k}, t_{k}\right)\left(\hat{S}=\hat{X}_{m, m \pm 1}^{(n)}, \hat{Y}_{n, n \pm 1}^{(m)}\right)$. This has a general analytical form [Eq. (25)] with the Melnikov functions $M_{\alpha, \alpha \pm 1}^{(\beta)}\left(t_{0}\right)(\alpha, \beta=m, n)$,

$$
M_{\alpha, \alpha \pm 1}^{(\beta)}\left(t_{0}\right)=-\frac{\partial}{\partial t_{0}} \int_{-\infty}^{\infty} H_{1}\left[q_{s}(t), p_{s}(t), t\right] d t,
$$

where integration is taken along the unperturbed separatrix connecting the saddle point $\left(x_{m}, y_{n}\right)$ with $\left(x_{m \pm 1}, y_{n}\right)(\alpha$ $=m, \beta=n)$ or with $\left(x_{m}, y_{n \pm 1}\right)(\alpha=n, \beta=m)$. The time shift $\Delta t(H)$ in Eq. (25) is equal to $T(H) / 8$, where $T(H)$ is unperturbed period of motion [Eqs. (19) and (20)] in each cell. Therefore, the separatrix maps for the system are

$$
\begin{gathered}
H_{k+1}=H_{k}+\epsilon M_{\alpha, \alpha \pm 1}^{(\beta)}\left(\varphi_{k}+\frac{\nu}{4 \pi} \ln \frac{2}{\pi\left|H_{k}\right|}+\chi\right), \\
\varphi_{k+1}=\varphi_{k}+\frac{\nu}{4 \pi}\left[\ln \frac{2}{\pi\left|H_{k}\right|}+\ln \frac{2}{\pi\left|H_{k+1}\right|}\right] .
\end{gathered}
$$

Each of these maps is invariant with respect to transformation (28), with the rescaling parameter $\lambda=\exp \left(4 \pi^{2} / \nu\right)$.

Consider, for example, the generating functions for perturbation function (21):

$$
\begin{aligned}
G_{\alpha, \alpha \pm 1}^{(\beta)}\left(t_{0}\right)= & -\frac{\epsilon}{2 \pi} \int_{-\infty}^{\infty}\left[a_{y} \sin (2 \pi y(t)-\nu t-\chi)\right. \\
& \left.-a_{x} \sin (2 \pi x(t)-\nu t-\chi)\right] d t
\end{aligned}
$$

Using the unperturbed trajectories along separatrices (18), and integrating Eq. (42), one can obtain the following expressions for the Melnikov functions in map (41):

$$
\begin{gathered}
M_{m, m \pm 1}^{(n)}\left(t_{0}\right)=(-1)^{n} a_{x} K^{( \pm)} \cos \left(\nu t_{0}+\chi\right), \\
\text { for } m+n=2 k, \\
M_{n, n \pm 1}^{(m)}\left(t_{0}\right)=(-1)^{m} a_{y} K^{( \pm)} \cos \left(\nu t_{0}+\chi\right) \\
\text { for } m+n=2 k+1,
\end{gathered}
$$

where

$$
K^{( \pm)}=\frac{\nu}{2 \pi} \frac{\exp ( \pm \nu / 4)}{\sinh (\nu / 2)}
$$

$(k=0, \pm 1, \pm 2, \ldots)$. From Eq. (43) it follows that all eight generating functions $G_{\alpha, \alpha \pm 1}^{(\beta)}\left(t_{0}\right)$ are not independent. Because of symmetry of perturbation (22), we have

$$
\begin{gathered}
M_{m, m \pm 1}^{(n)}\left(t_{0}\right)=-M_{m \mp 1, m}^{(n+1)}\left(t_{0}\right), \\
M_{n, n \pm 1}^{(m)}\left(t_{0}\right)=-M_{n \mp 1, n}^{(m+1)}\left(t_{0}\right) .
\end{gathered}
$$

The existence of a rescaling invariance of motion near saddle points (23) and (24), studied in Sec. II C by numerical integration of the equations of motion, is due to these symmetries. Using the separatrix maps (41) one can prove this rescaling property. This is shown in the Appendix by constructing maps $\hat{F}_{m n}^{(p)} \quad(p=1,2,3,4)$ for fixed points $\left(\varphi_{q, s}, H_{q, s}\right)$ near the saddle points $\left(x_{m}, y_{n}\right)$ at each branch $p$ of section $\Sigma_{m n}$, i.e.,

$$
\left(\varphi_{q, s}+2 \pi s, H_{q, s}\right)=\left(\hat{F}_{m n}^{(p)}\right)^{q}\left(\varphi_{q, s}, H_{q, s}\right),
$$

similar to those maps $\hat{F}_{p}$ introduced to determine the fixed points [Eqs. (33)] in the model studied in Sec. III A.

Using separatrix maps, we have plotted Poincaré sections of orbits at $\Sigma_{00}$ and $\Sigma_{01}$, shown in Fig. 11 for the same parameters as in Fig. 6(a): $\epsilon_{a}=0.0208$ and $\chi_{a}=0$. The rescaling parameter $\lambda=\exp \left(4 \pi^{2} / \nu\right)=16$. Corresponding plots 

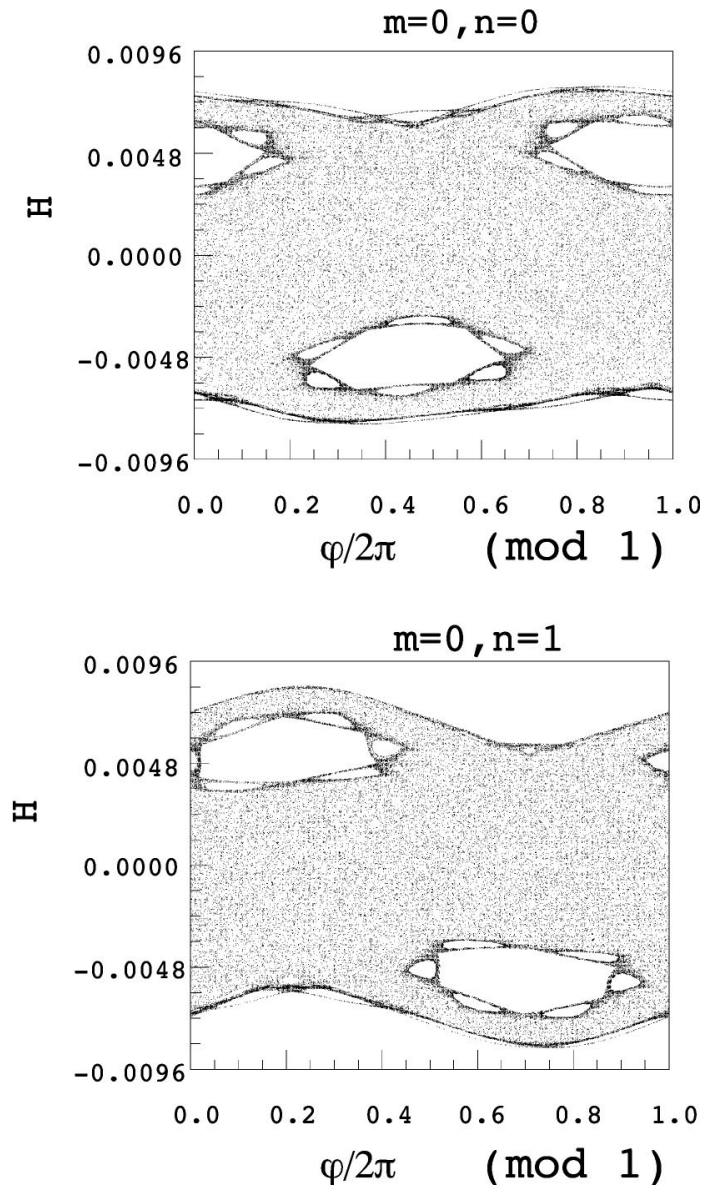

FIG. 11. Poincare sections of orbits of the periodic vortical flow [Eqs. (16) and (21)] obtained by separatrix mapping at sections $\Sigma_{00}$ and $\Sigma_{10}$ for the parameters $\epsilon_{a}=0.0208$ and $\chi_{a}=0$. The rescaling parameter $\lambda=\exp \left(4 \pi^{2} / \nu\right)=16$. Other parameters are $a_{x}=1$ and $a_{y}$ $=0.5$.

at sections $\Sigma_{11}$ and $\Sigma_{10}$ may be obtained from Poincare plots at $\Sigma_{00}$ and $\Sigma_{10}$ by shifting the phase $\varphi$ by $\pi$, respectively. Similar plots for the rescaled parameters $\epsilon_{b}=\lambda^{-1 / 2} \epsilon_{a}$ $=0.0052$ and $\chi_{b}=\chi_{a}+\pi / 2$ are presented in Fig. 12 at sections $\Sigma_{01}$ and $\Sigma_{11}$. Note that the axes $\varphi$ and $H$ in Fig. 12 are inverted. These figures confirm the rescaling invariance of motion with regard to transformations (23) and (24).

\section{STATISTICAL PROPERTIES OF CHAOTIC MOTION}

In this section and subsequent sections we study the statistical properties of chaotic motion in a stochastic layer, and their dependence on its structure. As noted above, the stochastic layers formed near the separatrix are not uniform. They consist of KAM stability islands embedded in a socalled stochastic sea. The structure of the stochastic layer is determined by the mutual positions and sizes of KAM islands. The existence of these islands leads to the deviation of chaotic motion from the normal diffusion processes because of long-time range correlations due to particles trapped near the islands. This process, known as anomalous transport, depends on the structure of the stochastic layer. Therefore, one can vary the transport properties by changing the structure of the stochastic layer. This may be done, for instance, by varying of the perturbation parameter $\epsilon$ or its frequency $\nu$.
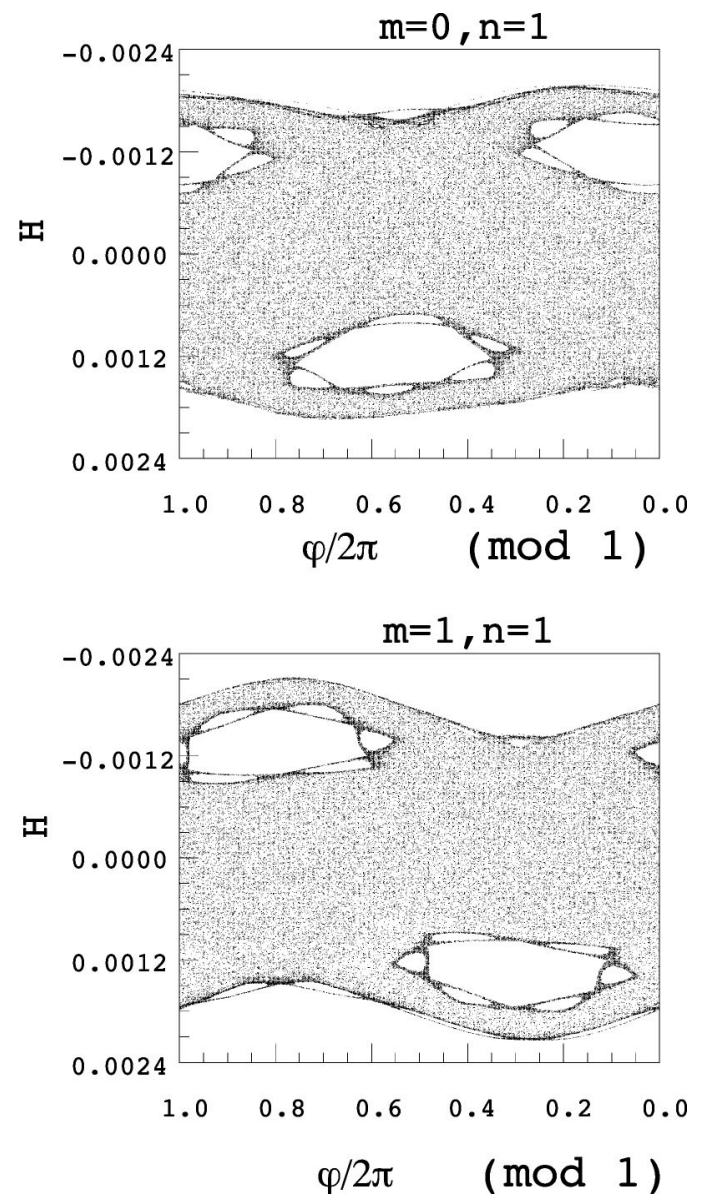

FIG. 12. The same as in Fig. 11, but at sections $\Sigma_{01}$ and $\Sigma_{00}$ for $\epsilon_{b}=\lambda^{-1 / 2} \epsilon_{a}=0.0052$ and $\chi_{b}=\chi_{a}+\pi / 2$. Other parameters are the same as in Fig. 11.

In the case of a stochastic layer formed near the separatrices, the chaotic transport is mainly determined by its structure near the saddle points, where particles spend more time than in other parts of the phase space. As shown in previous sections, the perturbed motion near the saddle points have a remarkable rescaling invariance with respect to the change of the perturbation amplitude $\epsilon$ and phase $\chi$ [Eqs. (6)] (for the fixed perturbation frequency $\nu$ ), i.e., the topology of the stochastic layer near the saddle points is a periodic function of $\ln \epsilon$ with period $\ln \lambda$. Therefore, one can expect that by varying $\epsilon$ one can periodically change the transport properties of chaotic motion in a stochastic layer. Below, we demonstrate these properties for the models of Hamiltonian systems considered in the previous sections.

\section{A. Mean residence time}

We first study a particle motion in a double-well potential subjected to the time-periodic perturbation described by Hamiltonian (10). This problem has a direct application in magnetic field line dynamics in divertor tokamaks [38], in chemistry, etc. Suppose that in the absence of perturbation a particle is trapped in one of the wells. A motion of particle may be described in the $(q, p)$ plane by the closed curve 1 in Fig. 1(a), and it is separated from the other well by the separatrix (curve 2). A time-periodic perturbation destroys the separatrix, replacing it by a stochastic layer. If the initial 


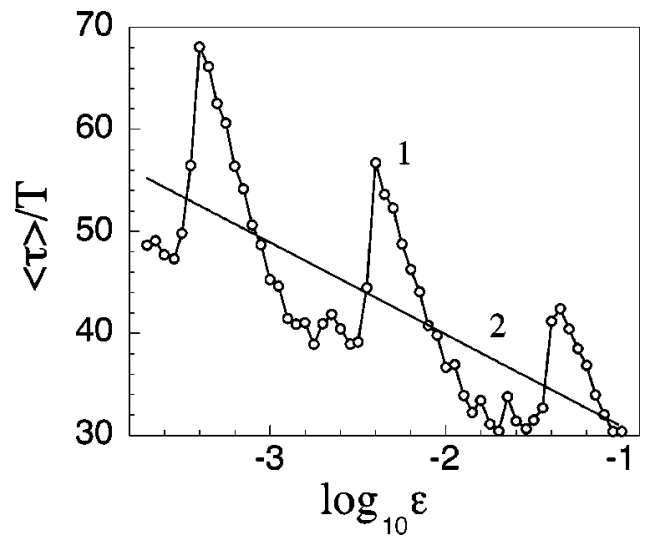

FIG. 13. A mean residence time $\langle\tau\rangle$ in the potential well normalized to the perturbation period $T_{0}$ versus a perturbation parameter $\epsilon$ (curve 1). Curve 2 describes the fitting of $\langle\tau\rangle$ by a linear function of $\log _{10} \epsilon: a-b \log _{10} \epsilon(a=21.87 \pm 2.64, b=3.92 \pm 0.462)$. The rescaling parameter $\lambda=10$.

orbit of the particle was inside the stochastic layer, it leaves the potential well during a certain time $\tau$. A residence time $\tau$ is a random number, and its statistics depends on the structure of the stochastic layer. Below we study the dependence of the mean residence time on the perturbation amplitude $\epsilon$.

Let us first estimate the qualitative dependence of the mean residence time $\langle\tau\rangle$ on the perturbation parameter $\epsilon$. If one does not take into account particles trapped by islands one could expect that $\langle\tau\rangle$ is proportional to the period of particle's orbit $T\left(H_{s}\right)$ inside the stochastic layer with an effective energy $H_{s}$, i.e., $\langle\tau\rangle \sim T\left(H_{s}\right)$. The effective energy $H_{s}$ is of order of the width of the stochastic layer $w_{s}(\epsilon)$ (supposing that at the separatrix $H=0$ ). Since $w_{s}(\epsilon) \sim \epsilon$, and taking into account the logarithmic asymptotics of the period $T\left(H_{s}\right)$ [Eq. (13)], one can obtain the dependence $\langle\tau\rangle \sim a-b \log _{10} \epsilon$, where $a$ and $b$ are independent of $\epsilon$ constants. Therefore neglecting the particle's trapping by islands one expects that the mean residence time linearly decreases with $\log \epsilon$.

Direct calculations of $\langle\tau\rangle$ were performed using the separatrix map [Eqs. (31)], and is presented in Fig. 13. The value of the perturbation frequency $\nu$ is chosen to have the rescaling parameter $\lambda=\exp (2 \pi / \nu)=10$. Averaging is made over $N=10^{6}$ orbits. Curve 1 describes a dependence on $\langle\tau\rangle$ on $\epsilon$, and curve 2 corresponds to its fitting with the linear-log law $\langle\tau\rangle \sim a-b \log _{10} \epsilon$. From the figure one can clearly see that the mean residence time does not depend monotonically on $\epsilon$. This shows that there are strong periodic oscillations around the linear-log dependence. These oscillations are due to a periodical variation of the topology of the phase space near the saddle point with the change of the perturbation amplitude $\epsilon$. The period of oscillations are determined by setting the rescaling parameter $\lambda$, i.e., equal to $\log _{10} \lambda$.

\section{B. Statistics of Poincaré recurrences}

One of the important statistical characteristics of the dynamically chaotic system is the statistics of Poincaré recurrences $P_{r e c}(\tau)$. This is defined as $N(\tau) / N$, where $N(\tau)$ is a number of recurrences with $t>\tau$, and $N$ is a full number of recurrences. This is related to the correlation function of dynamical variables $C(\tau)=\langle\dot{x}(t+\tau) \dot{x}(t)\rangle$ (see, e.g., Ref. [31]),

$$
C(\tau) \sim \tau P_{\text {rec }}(\tau) /\langle\tau\rangle,
$$

where $\langle\tau\rangle$ is the mean recurrence time. The diffusion coefficient (rate) $D$ is directly related to the correlations,

$$
D \sim \int_{0}^{\infty} C(\tau) d \tau \text {. }
$$

Asymptotically, probability $P_{r e c}(\tau)$ decays exponentially with $\tau$ in the case of fully developed chaos [3]. Numerous studies of $P_{r e c}(\tau)$ show that, for systems with partially chaotic regions, the recurrence has a power law $P_{\text {rec }}(\tau) \sim \tau^{-p}$ at a large time. The first calculations of the exponent $p$ for the separatrix map and other different maps gave $p \approx 1.5[31,30]$. During the last decade values of $p \approx 1-2.5$ have been found for different Hamiltonian systems [31,33,48-50]. However, Chirikov presented some arguments that the value of $p$ should be equal to 3 [51], which is strongly different from $p \approx 1.5$. Murray maintained that in order to achieve the exponent $p=3$, one requires larger times [52]. Recently a power-law decay $P_{r e c}(\tau) \sim \tau^{-p}$ with $p=3$ was numerically observed at very large times for dynamical chaos in a standard map with the critical golden KAM invariant curve, i.e., $m: n=\nu / \omega=(\sqrt{5}-1) / 2[53]$.

We have studied statistics of first return times to the fourth branch of section $\Sigma$ (Fig. 7) in the above considered double-well potential for different perturbation parameters $\epsilon$. The other parameters were the same as in Sec. IV A. All calculations are performed using separatrix map (31). The mean recurrence time $\langle\tau\rangle$ as a function of the perturbation parameter $\epsilon$ is shown in Fig. 14(a). Similar to the mean residence time (see Fig. 13), this is also a quasiperiodic function of $\log _{10} \epsilon$ with the period $\log _{10} \lambda$. However, the maxima of the mean residence time correspond to minima of the mean recurrence time $\langle\tau\rangle$, and vice versa.

The probability of recurrences $P(\tau)$ is computed up to moderate times $\tau \leqslant 10^{5} T$. This is shown in Fig. 14(b) for two different perturbation amplitudes $\epsilon=0.01$ (curve 1) and $\epsilon$ $=0.0126$ (curve 2), corresponding to the local minimum and maximum values of the mean recurrence time in Fig. 14(a), respectively. The straight lines correspond to the power-law decay $\tau^{-p}$. One can see from Fig. 14(b) that $P(\tau)$ decays oscillatingly near the power law $\tau^{-p}$, which was also observed in Ref. [31]. The amplitude of these oscillations varies with the perturbation parameter $\epsilon$. Estimations of the exponent $p$ for different $\epsilon$ were performed by a fitting of the probability of recurrences $P(\tau)$ with the power law $C \tau^{-p}$ in the time interval $10^{2} \leqslant \tau / T \leqslant 10^{5}$. The latter is of the order of the oscillation period of $P(\tau)$ around the power law $\tau^{-p}$. The dependence of the exponents $p$ on $\epsilon$ is shown in Fig. 14(c), from which one can recognize a periodic dependence of $p$ on $\log _{10} \epsilon$ with the period $\log _{10} \lambda=\log _{10} 10$. The values of $p$ vary between 1 and 2, and oscillate near the average value $p \approx 1.5$ which was observed in most previous calculations mentioned above.

\section{CHAOTIC TRANSPORT IN A STOCHASTIC LAYER. THREE-WAVE FIELD MODEL}

In this section we consider a chaotic transport of particles in a stochastic layer for the three-wave field model [Eq. (7)] 

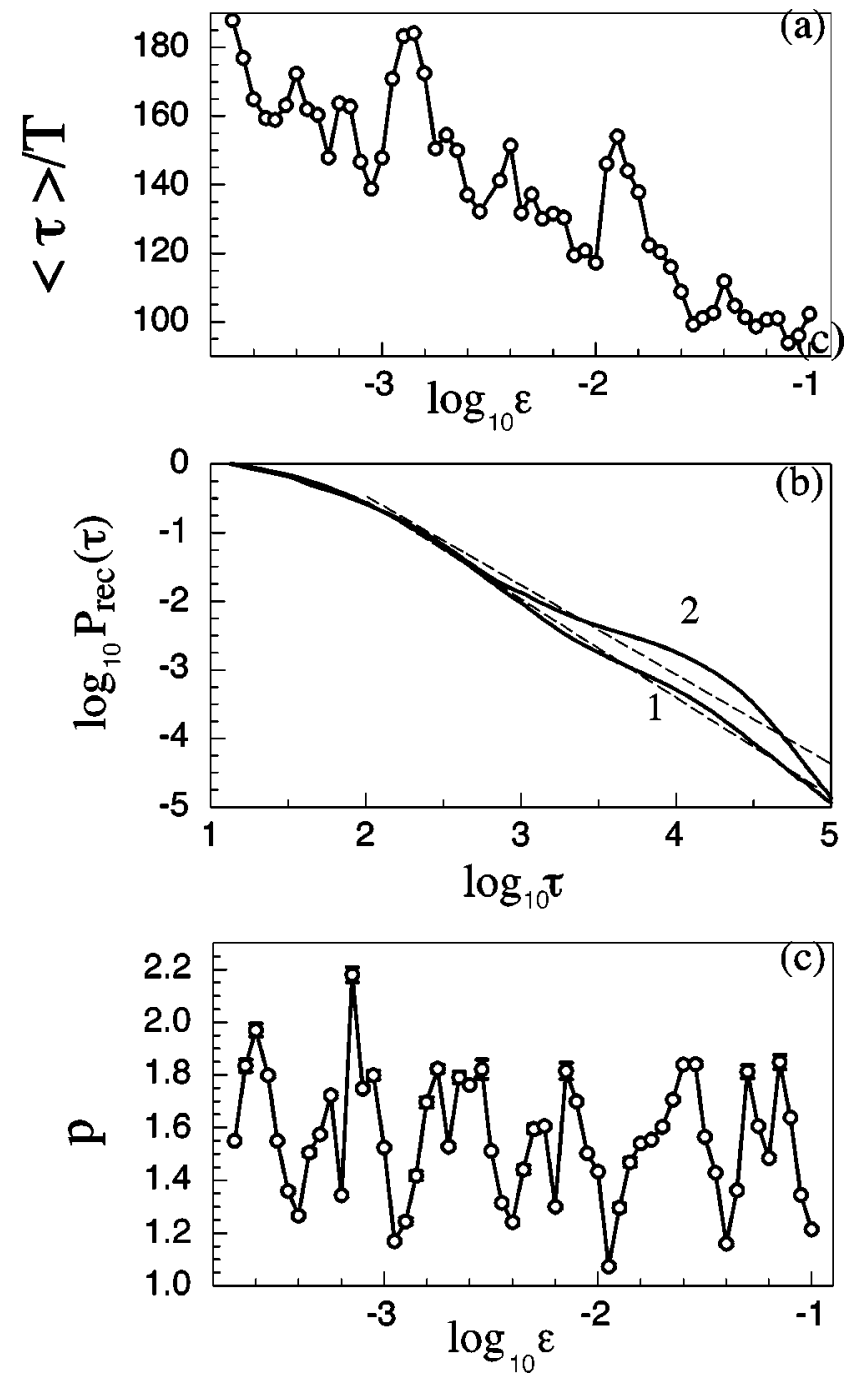

FIG. 14. (a) Average recurrence time $\langle\tau\rangle$ as a function of the perturbation amplitude logarithm, $\log _{10} \epsilon$. (b) Poincaré recurrence $P_{\text {rec }}(\tau)$ to the fourth branch of section $\Sigma$ (see Fig. 7) for the two perturbation amplitudes: $\epsilon=0.01$ (curve 1) and $\epsilon=0.0126$ (curve 2). (c) Exponents $p$ of asymptotics of $P_{r e c}(\tau) \sim \tau^{-p}$ as a function of $\log _{10} \epsilon$.

(see Sec. II A). We will study statistical properties of transport along the (infinite) $q$ axis, particularly advection and diffusion, by calculating the first, $\langle q\rangle$, and the second, $\sigma^{2}(t)=\left\langle(q-\langle q\rangle)^{2}\right\rangle$, moments of the spatial displacement, respectively, as well as the probability density function (PDF) $P(q, t)$ for a particle with position $q$ at time instant $t$ as a function of the perturbation amplitude $\epsilon$.

Calculations of the statistical moments were performed using direct numerical integrations of motion equations for the Hamiltonian (7). Since the numerical integration of the Hamiltonian system is rather time consuming, we have also exploited the fast numerical algorithm based on the separatrix map (38) to calculate statistical moments and the PDF.

For all calculations a perturbation frequency is chosen as $\nu=4.53236 \omega_{0}$. Then the rescaling parameter is $\lambda=4$. For simplicity we set $a_{+}=1$ and $a_{-}=0$ for the amplitudes of the perturbation waves to consider an advection. On the other hand we set $a_{+}=a_{-}=1$ to study "pure" diffusion. A numerical integration of the Hamiltonian system (7), was per-

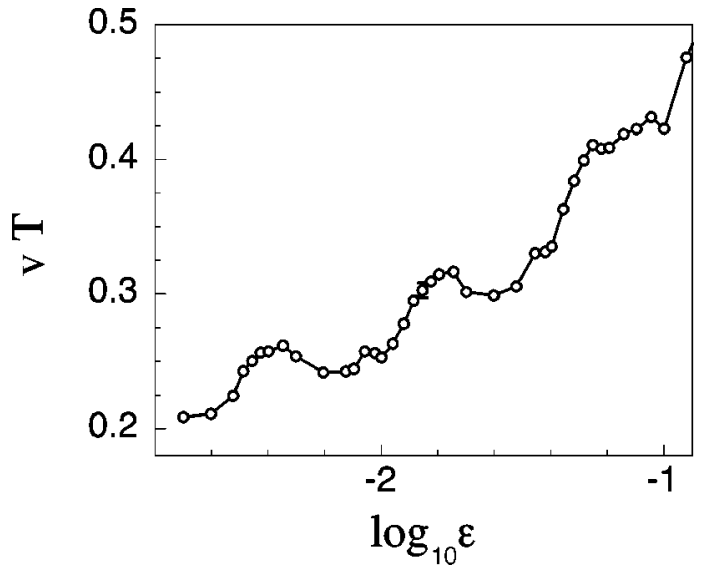

FIG. 15. Advection velocity $v$ vs the logarithm of the perturbation amplitude, $\log _{10} \epsilon$, as obtained by direct numerical integration. The rescaling parameter $\lambda=\exp \left(2 \pi \omega_{0} / \nu\right)=4$.

formed up to the time instant $t=2 \times 10^{4} T$ for different perturbation parameters $\epsilon$. Here $T=2 \pi / \nu$ is the period of the perturbation. A set of initial data at $t=0$, consisting of $N$ $=5 \times 10^{3}$ trajectories, was taken in a square region centered at a hyperbolic fixed point $(q=\pi, p=0)$.

\section{A. Advection}

An advection in the stochastic layer takes place in the direction of the perturbation wave with the larger amplitude $a_{\max }=\max \left(a_{+}, a_{-}\right)$. The maximum advection occurs if only one perturbation wave is present. We consider this case, setting $a_{+}=1$ and $a_{-}=0$. The mean value of the particle's coordinate $\langle q(t)\rangle$ is calculated by numerical integration of equations of motion for the different perturbation parameter $\epsilon$ in the interval $[0.002,0.4]$. This shows that at least up to $t \leqslant 2 \times 10^{4} T$ the mean coordinate $\langle q(t)\rangle$ is linear function of time $t$, i.e., $\langle q(t)\rangle=v t$, with an advection speed $v$. However, the advection speed $v$ is not a monotonic function of perturbation parameter $\epsilon$. Similar to the mean residence time studied in Sec. IV, it varies quasiperiodically with the change of $\log \epsilon$ as shown in Fig. 15. Its period is equal to $\log _{10} \lambda$.

\section{B. A second moment of spatial displacement}

The second moments $\sigma^{2}(t)$ of the spatial displacement are calculated for the $a_{+}=a_{-}=1$. Because of the fact that the perturbation in Eq. (7) in this case acts symmetrically on particles traveling in both positive and negative directions along the $q$ axis, and gives rise to no advection, i.e., the mean value of $q$ is expected to be $\langle q\rangle=0$.

The dependence of $\sigma^{2}(t)$ on the perturbation amplitude $\epsilon$ is shown in Fig. 16 at two different time instants: curve 1 corresponds to $t=10^{4} T_{0}$, and curve 2 to $t=2 \times 10^{4} T_{0}$. The thick curves describe the results obtained from a numerical integration of the equations of motion, while the thin curves corresponds to calculations by the separatrix map (38) (with an average over $N=10^{4}$ orbits). One can see that the separatrix map (38) correctly reproduces the results of direct numerical integrations with a good accuracy up to the value $\epsilon$ $=0.1$. Figure 16 clearly shows the strong quasiperiodical dependence of the second moment $\sigma^{2}(t)$ on the perturbation parameter $\epsilon$. There are local maxima of $\sigma^{2}(t)$ at the values 


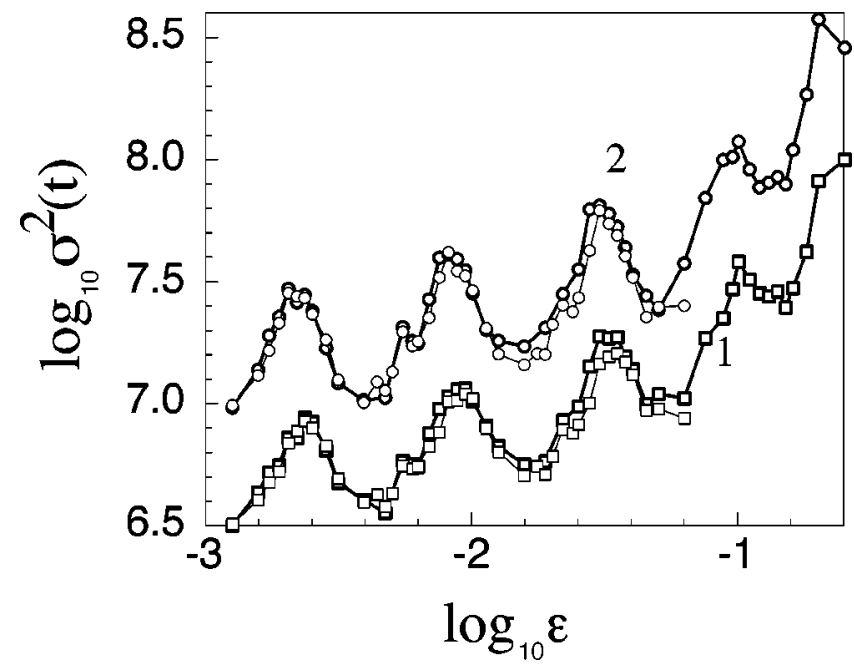

FIG. 16. Second moment $\sigma^{2}(t)$ vs $\log _{10} \epsilon$ as obtained by direct numerical integration. Solid curve 1 corresponds to $t=10^{4} T_{0}$, and solid curve 2 to $t=2 \times 10^{4} T_{0}$ (thick lines). The corresponding thin line curves describe the results obtained by the separatrix map [Eq. (38)].

$\epsilon_{\max }^{(j)}=\lambda^{-j} \epsilon_{\max }$ and $\boldsymbol{\epsilon}_{\max } \approx 0.192$, and local minima at $\boldsymbol{\epsilon}_{\min }^{(j)}$ $=\lambda^{-j} \epsilon_{\text {min }}$ and $\epsilon_{\text {min }} \approx 0.08(j=1,2, \ldots)$. For large perturbation amplitudes $\epsilon>0.1$ the quasi-periodical behavior of $\sigma^{2}(t)$ is less pronounced since the rescaling property of Hamiltonian system starts to violate for large perturbations.

The behavior of the second moment $\sigma^{2}(t)$ for long times $t>2 \times 10^{4}$ was studied using separatrix map (38). Figure 17 shows $\sigma^{2}(t)$ versus $\epsilon$ at the different time instants $t / T_{0}$ $=10^{4}, 2 \times 10^{4}, 5 \times 10^{4}, 10^{5}$, and $10^{6}$, continuously numbered $1-5$, respectively. With increasing time, the periodic dependence of $\sigma^{2}(t)$ on $\epsilon$ becomes more pronounced.

The profiles near the maxima become sharper, with shallow regions between them. The positions of the maxima $\epsilon_{\max }^{(j)}$ move toward smaller $\epsilon$, but the positions of the minima $\epsilon_{\min }^{(j)}$ become less distinct. The sharp maxima are due to the crossing the critical perturbation amplitude $\epsilon_{m n}^{(-)}$for destruction of

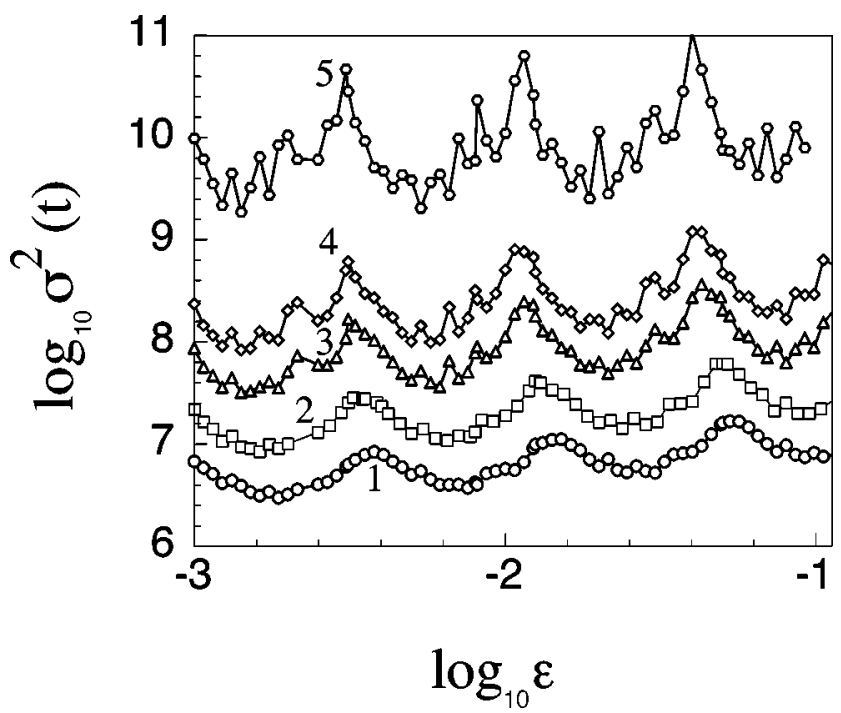

FIG. 17. Same as in Fig. 16, but for the time instants $t$ $=10^{4} T_{0}(1), 2 \times 10^{4} T_{0}(2), 5 \times 10^{4} T_{0}(3), 10^{5} T_{0}$ (4), and $10^{6} T_{0}(5)$.

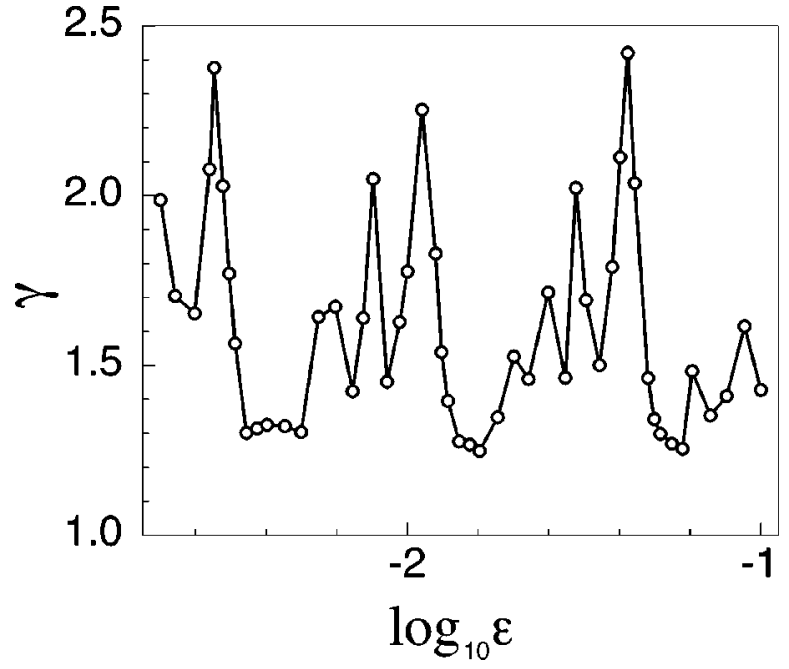

FIG. 18. Exponent $\gamma$ vs the logarithm of the perturbation amplitude, $\log _{10} \epsilon$. It is obtained by fitting $\sigma^{2}(t)$ with $2 D t^{\gamma}$ in the large time interval $10^{4} T_{0} \leqslant t \leqslant 10^{5} T_{0}$.

the last KAM invariant curve between the stochastic layer and the $m: n$ resonance of type 1 (islands of type 1 in Fig. 3), i.e., $m \omega\left(h_{m n}\right)=n \nu\left(h_{m n}<0\right)$, with the smallest possible number $m$. Just before crossing the invariant curve, more orbits are trapped by islands of types 2 and 3 . The reason is that the corresponding critical perturbation amplitude $\epsilon_{m n}^{(+)}$ for these resonances $\left[m \omega\left(h_{m n}\right)=n \nu, h_{m n}>0\right]$ is smaller than for the resonance of type 1, i.e., $\boldsymbol{\epsilon}_{m n}^{(+)}<\boldsymbol{\epsilon}_{m n}^{(-)}$. This asymmetry is due to the correction term $O(h)$ in Eq. (8). This results in an enhancement of the transport rate along the $q$ axis. After crossing the critical $\epsilon_{m n}^{(-)}$, the resonance $m: n\left(h_{m n}<0\right)$ joins the stochastic layer, and orbits begin to be trapped by that resonance. This leads to the decreasing of the transport rate. The small amplitude oscillations in $\sigma^{2}(t)$ with respect to $\epsilon$ are due to the joining of $m: n$ resonances with higher numbers $m$. The long time evolution more precisely reveals the existence of critical perturbation amplitudes $\epsilon_{m n}^{( \pm)}$for destruction of KAM invariant curves between the stochastic layer and the $m: n$ resonance.

For large times $t$ the asymptotics is $\sigma^{2}(t) \sim t^{\gamma}$. The exponent $\gamma$ is also a strong quasiperiodic function of $\ln \epsilon$ with the period $\ln \lambda$. The dependence $\gamma$ on $\epsilon$ obtained using the separatrix map (38) is shown in Fig. 18. The chaotic transport along the $q$ axis is superdiffusive $(\gamma>1)$ for all perturbation amplitudes. The exponent $\gamma$ takes maximum and minimum values at the same $\epsilon$ values as $\sigma^{2}(t)$ does. Regions with $\gamma$ $>2$ correspond to the acceleration regimes.

\section{Probability density function}

Separatrix map (38) is also applied to calculate the PDF $P(q, t)$. The PDF $P(q, t)$ was calculated at the time instant $t=2 \times 10^{4} T_{0}$ for perturbation parameters $\epsilon$ in the interval $[0.002,0.1]$. The number of orbits is $N=10^{5}$. The PDF is almost symmetric with respect to $q=0$, and it is mostly localized near this point. The width $2 \Delta \sigma$ of $P(q, t)$, defined as an area $-\Delta \sigma<q<\Delta \sigma$ where half the orbits are localized, i.e., $\int_{-\Delta \sigma}^{\Delta \sigma} P(q, t) d q=0.5$, also has a strong periodical dependence on $\ln \epsilon$ similarly to $\sigma^{2}(t)$. 

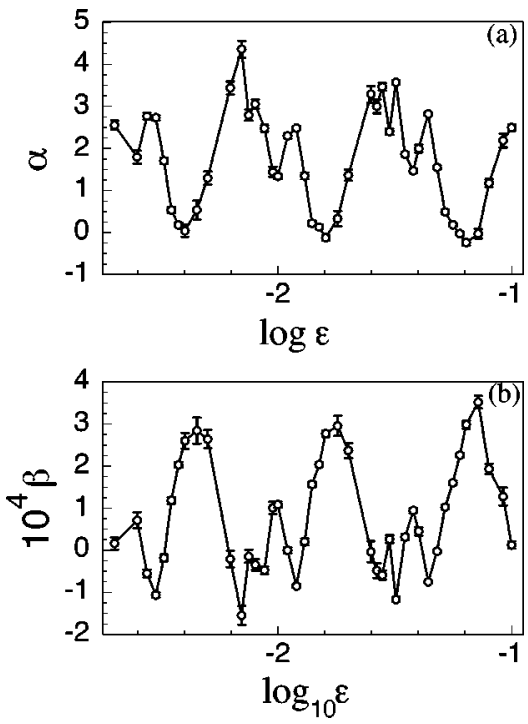

FIG. 19. Fitting parameters $\alpha$ and $\beta$ for a power exponential law $P(q, t) \sim|q|^{-\alpha} \exp (-\beta|q|)$ : (a) $\alpha$ versus $\epsilon$; (b) $\beta$ versus $\log _{10} \epsilon$. A time instant $t=2 \times 10^{4} T_{0}$.

One should note that the second moment $\sigma^{2}(t)$ and the width $\Delta \sigma$ describe the width of the probability density functions. However, in the case of anomalous (non-Gaussian) transport they describe different physical situations of the transport process. The width $\Delta \sigma$ describes the PDF near the central part where half of the particles are located. The main contribution to $\Delta \sigma$ comes from random particles and particles trapped by islands due to stickiness. On the other hand, contributions to $\sigma^{2}(t)$ mainly comes from particles with long distance flights. In the case of normal Gaussian transport both $\sigma^{2}(t)$ and $\Delta \sigma$ would have the same physical nature.

The main feature of $P(q, t)$ is its long tail asymptotics for $|q| \gg \Delta \sigma$. The latter depends significantly on the perturbation parameter $\epsilon$. The comparison, for instance, of the two PDF's at $\epsilon=0.048$ and 0.08 , for which the second moments $\sigma^{2}(t)$ have maximum and minimum values, respectively, shows that while the PDF for $\epsilon=0.048$ has a slowly decaying tail, the PDF for $\epsilon=0.08$ decays much more quickly. We have approximated $P(q, t)$ asymptotically by power exponential law $P(q, t) \sim|q|^{-\alpha} e^{-\beta|q|}$. The fitting exponents $\alpha$ and $\beta$ at the time instant $t=2 \times 10^{4} T_{0}$ are presented in Fig. 19: (a) shows $\alpha$ versus $\epsilon$, while (b) shows $\beta$ versus $\epsilon$. First a strong quasiperiodic dependence of these parameters on $\log _{10} \epsilon$ appears, with the period $\log _{10} \lambda$ similar to that for the exponent $\gamma$ in the time asymptotics of $\sigma^{2}(t)$.

There are regions of $\epsilon$ at the minima of $\sigma^{2}(t)$, where the parameter $|\beta|$ is relatively small, and the tail of the PDF is more closely described by the powerlike law $|q|^{-\alpha}$. At growing phases of the quasiperiodical dependence of $\sigma^{2}(t)$ on $\epsilon$, the parameter $\beta$ may take even negative values, which means that the tail of the PDF decays even more slowly than the powerlike law $|q|^{-\alpha}$ (see Fig. 16). On the other hand, the exponential decrease $e^{-\beta|q|}$ of $P(q, t)$ prevails in regions of $\epsilon$ where $\sigma^{2}(t)$ is decreasing. The exponential decrease of the probability to find particles at large $q$ is due to the trapping of particles by the $m: n$ resonance with the smallest possible number $m$ (the island of type 1 in Fig. 3) (when $\epsilon$ crosses the critical value $\epsilon_{m n}^{(-)}$for destruction of the last KAM invariant curve between the stochastic layer and the $m: n$ resonance). On the other hand, for $\epsilon<\epsilon_{m n}^{(-)}$, particles are trapped by resonances of types 2 and 3 , and therefore they can travel long distances $q$. In this case, the tail of $P(q, t)$ decays more slowly than $|q|^{-\alpha}$. Overall the results show that the asymptotics of $\operatorname{PDF} P(q, t)$ for $|q| \gg \Delta \sigma$ depends significantly on the structure of the stochastic layer, and it is mainly determined by the outermost KAM stability islands at the chaos border.

\section{CHAOTIC TRANSPORT IN 2D PERIODIC VORTICAL FLOW}

In this section we consider a chaotic transport in a stochastic web of the 2D time-dependent periodic vortical flow (16). For simplicity we consider a perturbation

$$
H_{1}(x, y, t)=\frac{\epsilon}{2 \pi}[\sin (2 \pi y)-\sin (2 \pi x)] \cos (\nu t+\chi),
$$

which may be considered as combination wave perturbations $\sin (2 \pi x-\nu t-\chi)+\sin (2 \pi x+\nu t+\chi) \quad$ and $\sin (2 \pi y-\nu t-\chi)$ $+\sin (2 \pi y+\nu t+\chi)$ propagating in opposite directions of the $x$ and $y$ axes, respectively. Thanks to symmetry property (22) of perturbation (48), there exists an additional rescaling invariance property [Eqs. (23) and (24)] of the system. Therefore, one expects that all statistical characteristics of the chaotic transport in such a system are to be quasiperiodical functions of the perturbation amplitude $\log _{10} \epsilon$ with the period $\log _{10} \lambda / 2$. For perturbation (48), one can expect that the chaotic transport along $x$ and $y$ directions are equivalent, in particular, mean spatial displacements $\langle x\rangle=\langle y\rangle=0$, and mean squared displacements $\left\langle x^{2}\right\rangle=\left\langle y^{2}\right\rangle$.

The statistical characteristics of the chaotic transport in a stochastic web were studied using the separatrix map constructed in Sec. III C. The separatrix map for perturbation (48) has the form of Eq. (41), with the Melnikov integral

$$
\begin{gathered}
M_{\alpha, \alpha \pm 1}^{(\beta)}\left(t_{0}\right)=(-1)^{\beta} K \cos \left(\nu t_{0}+\chi\right), \\
K=\frac{\nu}{4 \pi} \frac{1}{\sinh (\nu / 4)} .
\end{gathered}
$$

We have chosen the rescaling parameter $\lambda=\exp \left(4 \pi^{2} / \nu\right)$ $=16$. The statistics of transport is studied averaging over $N$ $=10^{4}$ orbits. In Fig. 20 a second moment of the radial displacement $\sigma_{r}^{2}(t)=\left\langle x^{2}(t)+y^{2}(t)\right\rangle$ is displayed as a function of the perturbation amplitude $\epsilon$ at the time instant $t=10^{4} T$. It shows a quasiperiodicity of $\sigma_{r}^{2}(t)$ with respect to $\log _{10} \epsilon$ with the period $\log _{10} \lambda / 2=\log _{10} 10 / 2$, which is twice as small as in cases of transport along the 1D stochastic layer studied in Sec. V. In Fig. 20 one can see also sharp periodic peaks of $\sigma^{2}(t)$ for some values of $\epsilon$. As we will see below they appear due to enhanced (superdiffusive) transport in the stochastic web.

The exponent $\gamma$ of asymptotics $\sigma_{r}^{2}(t) \sim t^{\gamma}$ determined by calculations up to $t \leqslant 10^{5} T$, are shown in Fig. 21. Similarly to $\sigma_{r}^{2}(t)$ it is periodically varying with $\log _{10} \epsilon$. From the figure one can see that there are large periodic intervals of the perturbation parameter $\epsilon$ where the values of $\gamma$ are less 


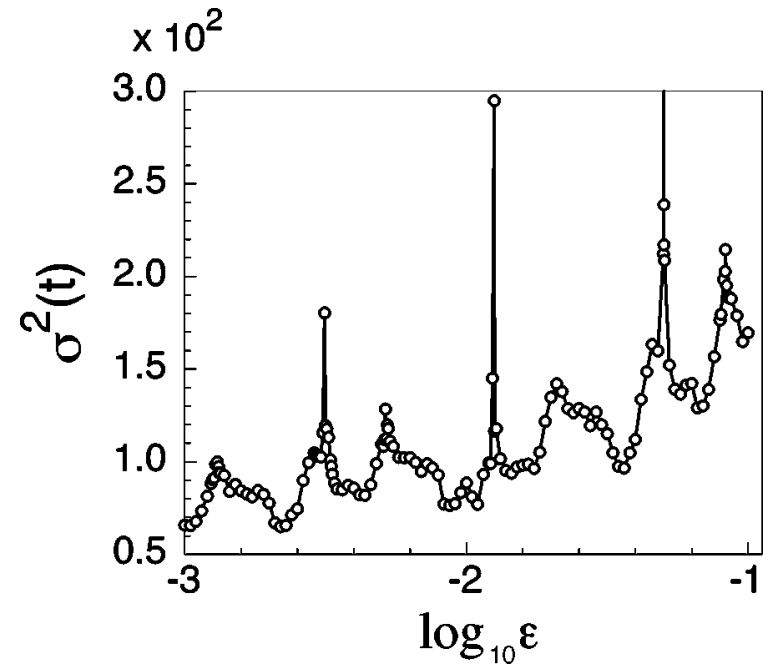

FIG. 20. Second moment of radial displacement $\sigma^{2}=\left\langle x^{2}+y^{2}\right\rangle$ versus $\log _{10} \epsilon$. A time instant $t=10^{4} T$. Rescaling parameter $\lambda$ $=\exp \left(4 \pi^{2} / \nu\right)=16$.

than but close to 1. Minima values of the exponent $\gamma$ are about 0.9 in small intervals of $\epsilon$ located near $\epsilon_{0} \lambda^{i / 2}\left(\epsilon_{0}\right.$ $=0.0022, i=0, \pm 1, \pm 2, \ldots)$. At values of $\epsilon$ the transport may be considered weakly subdiffusive.

For most values of $\epsilon$ the exponent $\gamma$ is close 1 , and the chaotic transport may be well approximated by the normal diffusion (Gaussian) process, and one can introduce a diffusion coefficient $D$ as $D_{\sigma}=\sigma_{r}^{2}(t) / 2 t(t \rightarrow \infty)$. The latter can be also found from the PDF $P(r, t)$, to find a particle with radial position $r=\sqrt{x^{2}+y^{2}}$ at a time instant $t$. In this case the diffusion coefficient $D$ is determined by the width of the radial Gaussian distribution $P_{G}(r, t)$ $=r\left(D_{G} t\right)^{-1} \exp \left[-r^{2} / 2 D_{G} t\right]$ if the initial distribution of particles at the time instant $t=0$ is the $\delta$ function, i.e., $P_{G}(r, 0)=2 \pi r \delta(r)$. For the normal diffusion process the diffusion coefficients $D_{\sigma}$ and $D_{G}$ determined by these two different way should be equal.

Diffusion coefficients calculated in such a way are presented in Fig. 22 as functions of $\epsilon$. Curve 1 describes the diffusion coefficient $D_{\sigma}$ determined from the mean squared

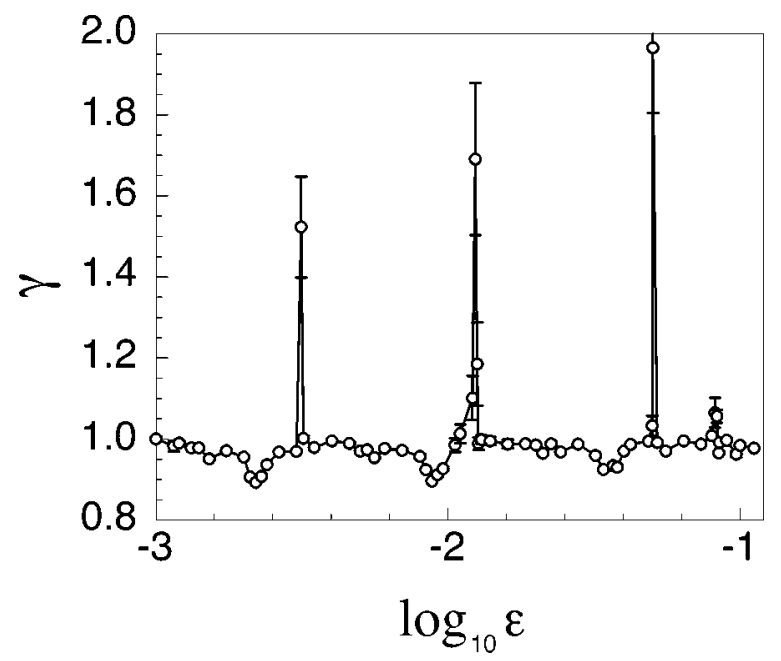

FIG. 21. Exponents $\gamma$ of the asymptotics $\sigma^{2} \sim t^{\gamma}$ versus $\log _{10} \epsilon$. The parameters of the system are the same as in Fig. 20.

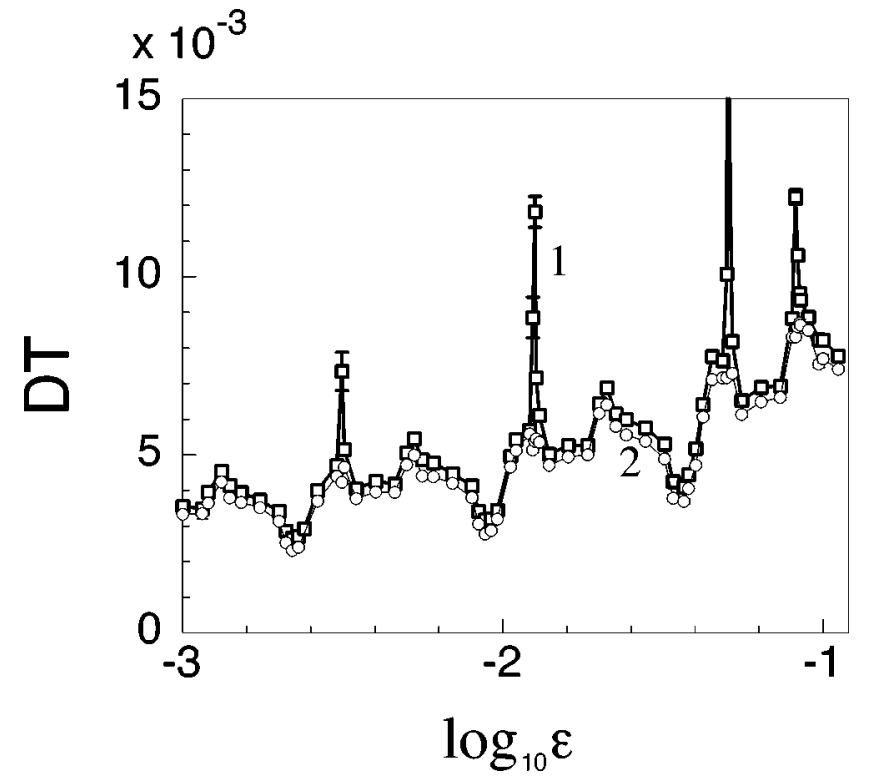

FIG. 22. Diffusion coefficients $D$ determined by the asymptotics $D_{\sigma}=\sigma^{2} / 2 t$ (curve 1) and by fitting with the radial Gaussian distribution $P_{G}(r, t)=r\left(D_{G} t\right)^{-1} \exp \left[-r^{2} / 2 D_{G} t\right]$ (curve 2) as functions of $\log _{10} \epsilon$. The parameters of the system are the same as in Fig. 20.

spatial radial displacement $\sigma_{r}^{2}(t)$ in the time interval $10^{4}$ $\leqslant t / T \leqslant 10^{5}$, and curve 2 corresponds to the $D_{G}$ obtained by fitting the numerically determined $\operatorname{PDF} P(r, t)$ at the time instant $t=10^{5} T$ with the radial Gaussian distribution $P_{G}(r, t)$. Figure 22 also shows a quasiperiodical dependence of both values of $D$ on $\log _{10} \epsilon$ similar to those ones in Figs. 20 and 21. For most values of $\epsilon$ the diffusion coefficients $D_{\sigma}$ and $D_{G}$ are close, but $D_{\sigma}$ systematically exceeds $D_{G}$. The reason for such a behavior was discussed in Sec. V C, and consists of the fact that there exists a difference between the second moment $\sigma_{r}^{2}(t)$ and the width of the PDF $P(r, t)$ in a case of anomalous transport. The coefficient $D_{G}$ is mainly determined by the central part of the PDF, while rare events with long flights may contribute to $D_{\sigma}$. Results shown in Fig. 22 suggest that in large periodic intervals of the perturbation parameter $\epsilon$, where differences between $D_{\sigma}$ and $D_{G}$ are small, the transport process may be considered a normal diffusion process.

\section{Superdiffusive regime}

From Fig. 22 it also follows that there are large differences between $D_{\sigma}$ and $D_{G}$ in the narrow periodic intervals of $\boldsymbol{\epsilon}$ located near values $\boldsymbol{\epsilon}=\lambda^{k / 2} \epsilon_{0}$ and $\boldsymbol{\epsilon}_{0}=0.0031$ $(k=0, \pm 1, \pm 2, \ldots)$. They correspond to the peaks in $\sigma_{r}^{2}(t)$ (see Fig. 20) and to the large exponents $\gamma$ in Fig. 21. For these values of $\epsilon$ the Gaussian approximation fails, and chaotic transport becomes superdiffusive. Enhanced transport is connected to the existence of flights of long distances (the Levý flight) at these values of $\epsilon$. A single event of flight or long distance is shown in Fig. 23 in the $(x, y)$ plane (a) and in the $(t, H)$ plane (b) for the specific value of $\epsilon=0.0124$ for which such a behavior occurs. These flights are connected with the stickiness of orbits to the specific KAM stability islands. They are shown in Fig. 24 on Poincaré sections of orbits on the $(x, y)$ plane near the two saddle points. (a) $x_{s}$ 


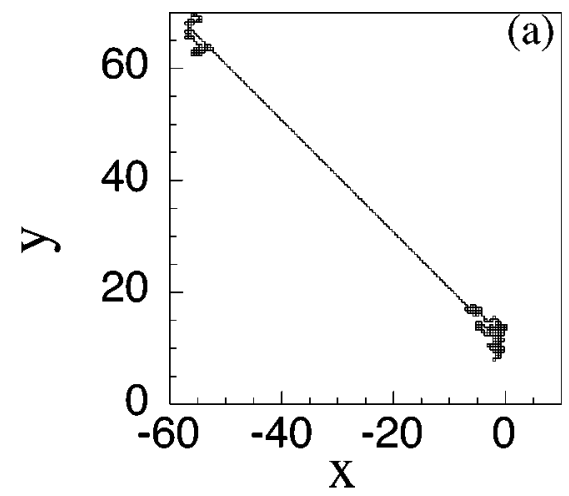

(b)

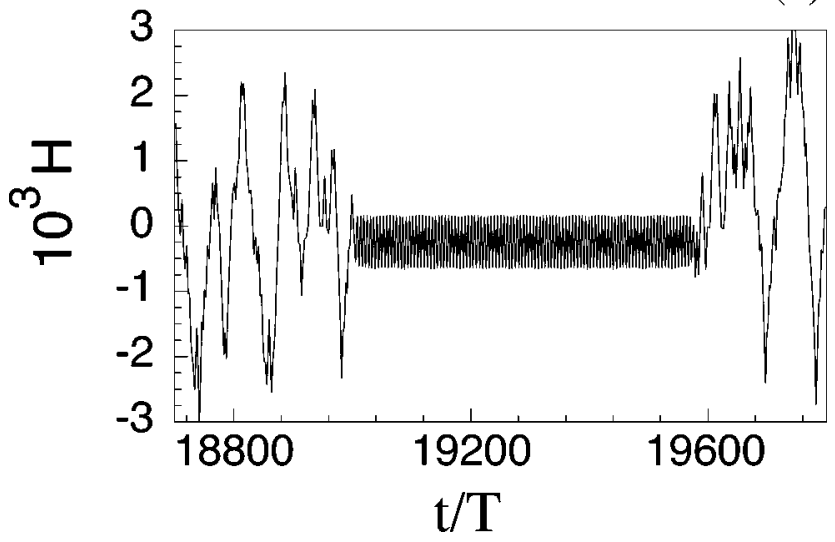

FIG. 23. Long distance flight event: (a) orbit in the $(x, y)$ plane; (b) orbit in the $(H, t)$ plane. Parameters are $\epsilon=0.0124$ and $\chi=0$.

$=-0.25, y_{s}=0.25$ and (b) $x_{s}=0.25, y_{s}=0.25$ obtained by direct integration of Hamiltonian equations using the BulirschStoer method mentioned in Sec. II C. Four types of islands (seen as dark sticks) responsible for flights of long distances are continuously labeled by $1-4$. Figure 24(c) shows a closeup view of the region near the first island. Islands 1-4 have different flight directions: the flight direction of the first island is $45^{\circ}$ with respect to positive direction of the $x$ axis, that for the second island is $315^{\circ}$, that for the third island is $225^{\circ}$, and that for the fourth island is $135^{\circ}$.

The structure of these tiny-sized KAM stability islands may be also shown in the $(t, H)$ plane by plotting Poincare plots in the $(t, H)$ plane using the separatrix map. These plots are presented in Fig. 25 (a) on section $\Sigma_{01}$ and (b) on section $\Sigma_{11}$. (c) shows a closeup view of the region near the tinysized KAM stability island shown in (b). The island with $H<0(H>0)$ in Fig. 25(a) corresponds to the first (second) and third (fourth) islands in Fig. 24(a), and the island with $H>0(H<0)$ in Fig. 25(b) corresponds to the first (second) and third (fourth) islands in Fig. 24(b), respectively. From positions $\left(x_{e}, y_{e}\right)$ of elliptic fixed points of islands in the $(x, y)$ plane, one can determine the values of energy $H_{e}$ for the fixed points of corresponding islands in the $(t, H)$ plane. From Fig. 24(a), it follows that for the first and third islands we have $H_{e}=H\left(x_{e}, y_{e}, t=k T\right) \approx-1.495 \times 10^{-4}$, and for the second and fourth islands we have $H_{e} \approx 1.4245 \times 10^{-4}$. Similarly, from Fig. 24(b) we have $\left|H_{e}\right|=6.4565 \times 10^{-4}$. These values reproduce with the good accuracy the corresponding values of $H$ determined by the separatrix map: $H_{e}$ $= \pm 1.48565 \times 10^{-4}$ at section $\Sigma_{01}$, and $H_{e}= \pm 6.50418$ $\times 10^{-4}$ at section $\Sigma_{11}$.

One should note that although the applied numerical integration scheme sufficiently well determines the positions of these island on the phase space $(x, y)$, it cannot resolve fine details of their structure because of a loss of accuracy. The fine structure of these tiny-sized KAM stability islands may be seen on Poincare plots in the $(t, H)$ plane, obtained by the separatrix map [see Fig. 25(c)].

First we should note the main specific feature of orbits trapped by islands responsible for flights. We call these flight islands. As one sees from Figs. 23(b), 24, and 25, the values of the energy variable $H$ for trapped orbits take successively positive and negative values near the saddle points; i.e., if $H_{k}>0$, then $H_{k+1}<0$ for every step of the separatrix map [Eqs. (41) and (49)]. The elliptic fixed points $\left(\varphi^{(e)}, H^{(e)}\right)$ of flight islands at any section $\Sigma_{m n}$ (or at equivalent sections $\left.\Sigma_{m \pm 2 q, n \pm 2 p}, q, p=1,2, \ldots\right)$ can be determined by the fixed points of the map $\hat{F}_{m n}^{(p)}$ [Eq. (45)], similar to those fixed points [Eqs. (33)] of KAM stability islands responsible for trapping in cells, but with the requirement that if $H_{k}^{(e)}>0$ then $H_{k+1}^{(e)}<0$, and vice versa. The maps $\hat{F}_{m n}^{(p)}$ are constructed similarly to Eqs. (A1) and (A2). For example, the map

$$
\hat{F}_{m, n}^{(2)}=\hat{Y}_{n+1, n+2}^{(m+2)} \hat{X}_{m+1, m+2}^{(n+1)} \hat{Y}_{n, n+1}^{(m+1)} \hat{X}_{m, m+1}^{(n)}
$$
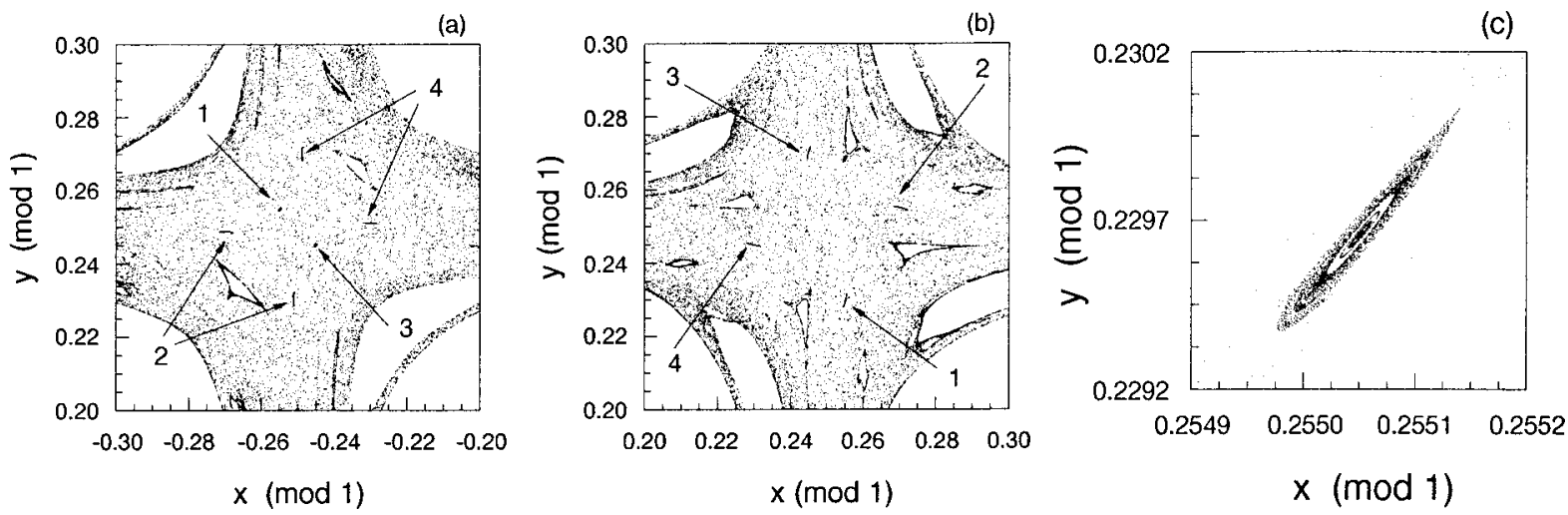

FIG. 24. Poincaré section near the saddle points: (a) $x_{s}=-0.25, y_{s}=0.25$; (b) $x_{s}=0.25, y_{s}=0.25$; four tiny-sized islands of regular motion responsible for long distance flights are shown by arrows 1-4. (c) Closeup view of the region near the first island shown in (b). Parameters are the same as in Fig. 23. 
(a)
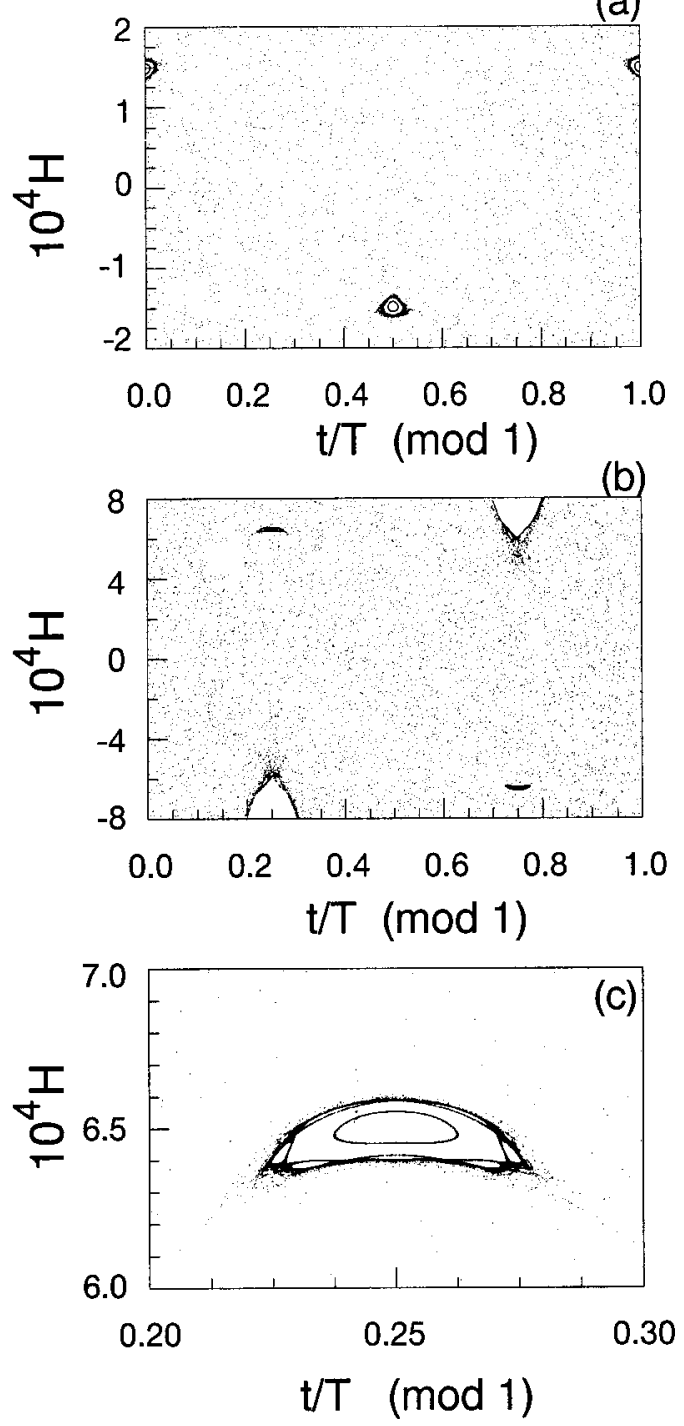

FIG. 25. Poincare plots obtained by the separatrix map in the $(H, t)$ plane at sections (a) $\Sigma_{00}$ and (b) $\Sigma_{10}$. The small-sized islands (with dark edges) are responsible for long distance flights. (c) Closeup view of one of the islands shown in (b). Parameters are the same as in Fig. 23.

transforms the fixed point of the first island at section $\Sigma_{m n}$ with even $m+n$ to an equivalent one at section $\Sigma_{m+2, n+2}$ (see Fig. 26).

To be specific, we determine the principal fixed points of the first island, corresponding to $q=1$. Using Eqs. (41) and (49), the equations for the fixed points $\left(\varphi^{(e)}, H^{(e)}\right)$ may be written

$$
\begin{gathered}
h_{2}=h_{1}+\epsilon K \cos w_{1}, \quad w_{2}=w_{1}+\Omega\left(h_{2}\right), \\
h_{3}=h_{2}-\epsilon K \cos w_{2}, \quad w_{3}=w_{2}+\Omega\left(h_{3}\right), \\
h_{4}=h_{3}-\epsilon K \cos w_{3}, \quad w_{4}=w_{3}+\Omega\left(h_{4}\right), \\
h_{1}=h_{4}+\epsilon K \cos w_{4}, \quad w_{1}+2 \pi s=w_{4}+\Omega\left(h_{1}\right),
\end{gathered}
$$

where $\Omega(h)=(\nu / 2 \pi) \ln (2 / \pi|h|), w=\varphi+\Omega(h) / 2+\chi$, and $s$ $=1,2, \ldots$ In Eq. (51) the following notations are introduced:

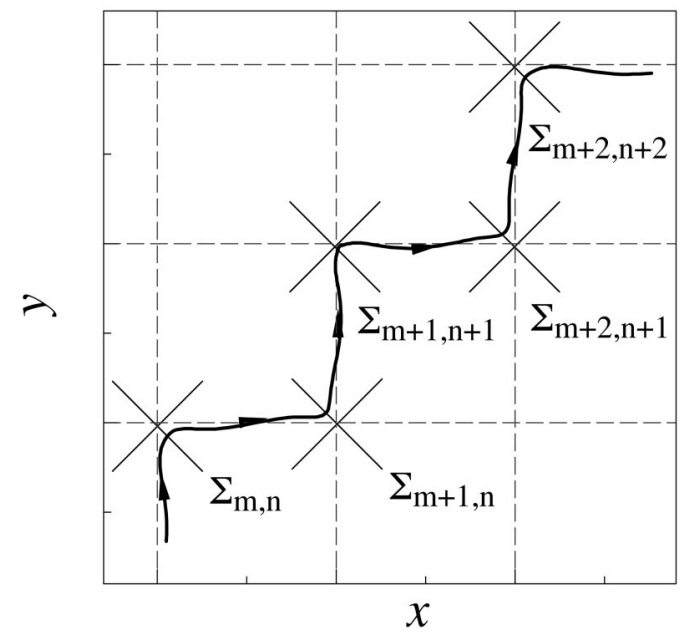

FIG. 26. Construction of the map $F_{m n}^{(2)}$ for the determination of fixed points of the first island responsible for flights.

$$
\begin{gathered}
\left(w_{1}, h_{1}\right)=(w, H) \text { at } \Sigma_{m, n} \text { and } \Sigma_{m+2, n+2}, \\
\left(w_{2}, h_{2}\right)=(w, H) \text { at } \Sigma_{m+1, n}, \\
\left(w_{3}, h_{3}\right)=(w, H) \text { at } \Sigma_{m+1, n+1}, \\
\left(w_{4}, h_{4}\right)=(w, H) \text { at } \Sigma_{m+2, n+1} .
\end{gathered}
$$

Fixed points of the first flight island should also satisfy the conditions $h_{1}<0, h_{2}>0, h_{3}<0$, and $h_{4}>0$. From the equations for the angular variables $w_{i}(i=1,2,3$, and 4$)$ in Eqs. (51), it follows that

$$
\sum_{i=1}^{4} \Omega\left(h_{i}\right)=2 \pi s \quad \text { or } \quad \prod_{i=1}^{4}\left|h_{i}\right|=16 /\left(\pi^{4} \lambda^{s}\right),
$$

where $\lambda$ is the rescaling parameter.

First we consider the cases when $h_{1}=h_{3}$ and $h_{2}=h_{4}$. In these cases Eqs. (51) may be reduced to

$$
\begin{gathered}
h_{2}=h_{1}+\epsilon K \cos w_{1}, \\
h_{1}=h_{2}-\epsilon K \cos \left[w_{1}+\Omega\left(h_{2}\right)\right], \\
\left|h_{1} h_{2}\right|=4 /\left(\pi^{2} \lambda^{s / 2}\right) .
\end{gathered}
$$

Taking into account that $h_{1}<0, h_{2}>0$, we come to the transcendental algebraic equation for $w_{1}$,

$$
\cos w_{1}-\cos \left\{w_{1}+\Omega\left[h_{2}\left(w_{1}\right)\right]\right\}=0,
$$

where $h_{2}\left(w_{1}\right)$ is a positive solution of the quadratic equation

$$
h_{2}^{2}-\epsilon K \cos w_{1} h_{2}+4 /\left(\pi^{2} \lambda^{s / 2}\right)=0 .
$$

Let us determine the fixed points of the island for the perturbation parameter $\epsilon$ near its specific value $\epsilon=0.0124$. For these values of $\epsilon$ the number $s$ is equal to 11. From Eqs. (54) and (55) we have numerically found that the fixed points $\left(\varphi^{(e)}, H^{(e)}\right)$ exist for $0.0121 \leqslant \epsilon \leqslant 0.031$. At section $\Sigma_{00}$ the phase $\varphi^{(e)}=\pi(\bmod 2 \pi)$, and $H^{(e)}$ changes in the interval $\left[-1.542623 \times 10^{-4},-7.518702 \times 10^{-5}\right]$, and at $\Sigma_{01}$ the phase $\varphi^{(e)}=3 \pi / 2(\bmod 2 \pi)$ and $6.263842 \times 10^{-4}$ 

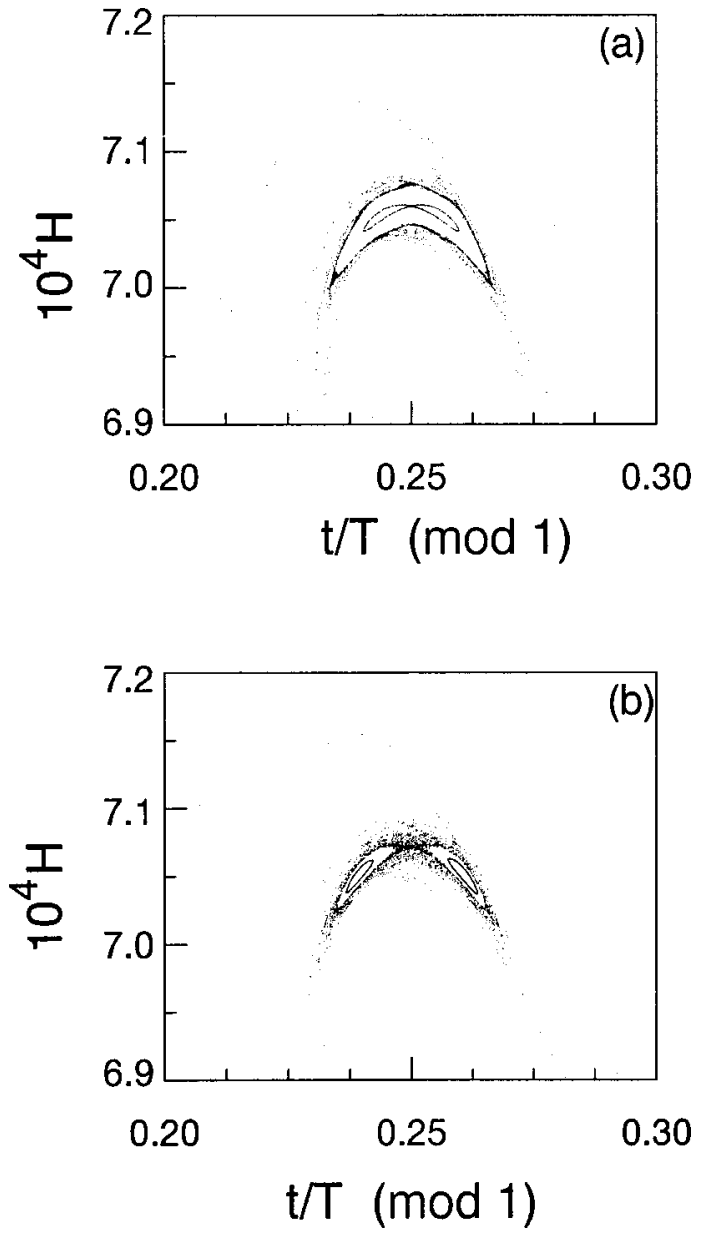

FIG. 27. The same as in Fig. 25(c) but for the perturbation parameters (a) $\epsilon=0.0132$ and (b) $\epsilon=0.01322$. Other parameters are the same as in Fig. 23.

$\leqslant H^{(e)} \leqslant 1.285161 \times 10^{-3}$, respectively. However, the fixed points are elliptic only for $0.0121 \leqslant \epsilon \leqslant 0.0131$, and are hyperbolic for $\epsilon>0.0131$. In the first case the eigenvalues $\lambda_{1,2}$ of the matrix

$$
\left[\begin{array}{ll}
\partial h_{2} / \partial h_{1} & \partial h_{2} / \partial w_{1} \\
\partial w_{2} / \partial h_{1} & \partial w_{2} / \partial w_{1}
\end{array}\right]
$$

are complex numbers, i.e., $\lambda_{1,2}=\exp ( \pm i \alpha)$, and in the second case $\lambda_{1,2}$ are real, i.e., $\lambda_{1}>1, \lambda_{2}<1, \lambda_{1} \lambda_{2}=1$.

A determination of flight islands with fixed points $h_{1}$ $\neq h_{3}$ or $h_{2} \neq h_{4}$ is more difficult. We have studied them by directly plotting Poincare sections in the $(\varphi, H)$ plane. Such islands appear for $\epsilon \geqslant 0.0132$ when the fixed point with $h_{1}$ $=h_{3}$ and $h_{2}=h_{4}$ becomes hyperbolic, and generates two elliptic fixed points. With increasing perturbation $\epsilon$, the stochastic layer near the separatrix grows. The islands disappear for $\epsilon>0.0133$. This process is shown in Fig. 27 by plotting Poincaré sections near these islands at section $\Sigma_{10}$ for two different perturbation parameters (a) $\epsilon=0.0132$ and (b) $\epsilon=0.01322$. The sizes of these islands is much smaller than ones with one elliptic point [compare with Fig. 25(c)].

Therefore, flights may occur for perturbation parameters $0.0121 \leqslant \epsilon \leqslant 0.0132$ due to the stickiness of the island with elliptic fixed points. Similar results can be obtained for the principal fixed points with $q=1$ of the second, third, and fourth islands. Fixed points with higher numbers $q \geqslant 2$ can be studied by a similar approach. However, this is a rather difficult task.

One can conclude that chaotic transport in a stochastic web of a 2D time-dependent periodic vortical flow may exhibit three types of stochastic processes: subdiffusive, normal Gaussian, and superdiffusive. Varying the perturbation parameter $\epsilon$, one can control the type of chaotic transport.

\section{CONCLUSIONS}

We have studied an important relationship between the structure of a stochastic layer and statistical properties of chaotic transport in it. It was shown that systems with topologically similar stochastic layers have similar statistical properties of transport. This property of dynamical systems was extensively studied in $1 \frac{1}{2}$-degree-of-freedom Hamiltonian systems. First we discussed the universal rescaling invariance of motion near the saddle points with respect to a rescaled transformation of the amplitude of a time-periodic perturbation with a shift of its phase; we also established an additional rescaling invariance of motion due to symmetries of the system. These properties give rise to periodic changes of the topology of the phase space near the saddle point with varying perturbation amplitudes. This leads, in turn, to quasiperiodical oscillations of statistical characteristics of transport with the change of the perturbation amplitude. The period of these oscillations, determining the rescaling parameter $\lambda=\exp (2 \pi \gamma / \nu)$, is a universal parameter which depends only on the expansion coefficient $\gamma$ of the unperturbed Hamiltonian near the saddle point, as well as on the frequency of the external perturbation $\nu$.

This effect is universal for a one-degree-of-freedom Hamiltonian system subjected to a small time-periodic perturbation regardless of the specific features of the system. One can expect the effect to occur in chaotic transport problems in structured flows-for instance, in chaotic mass transport in a chain of vortices in a shear layer [16]. Similarly, it may also be observed in models of physical systems which are described by a stochastic web (see, e.g., Ref. [33]).

Our study shows that the chaotic transport rate is not a monotonic function of the perturbation amplitude $\epsilon$, in spite of the fact that the stochastic layer's width increases linearly with $\epsilon$. This suggests, first, that the width of the stochastic layer, the determination of which was the primary goal of many works (see, e.g., Ref. [54], and references therein), does not completely characterize a chaotic motion. The existence of KAM stability islands embedded in a stochastic layer is one of its essential features, and particularly the outermost islands play a crucial role in chaotic transport. This situation is not taken into account by qualitative transport theories; for instance, quasilinear theory, predicts the monotonic dependence of transport rate on the perturbation amplitude.

The established effect also shows the possible range of controlling Hamiltonian chaos [55], in particular, chaotic transport, by varying the perturbation amplitude. The effect may be useful in controlling the transport of heat and particles in magnetic fusion devices with stochastic magnetic field lines [56], in controlling the transport of passive scalars 
in a chain of vortices [16], or in a mixing of fluids [7].

One should note that oscillations of the normal diffusion coefficient $D$ as a function of the stochasticity parameter $K(K>1)$, with a period $2 \pi$, were observed in standard (Chirikov-Taylor) mapping [2,57,58]. However, this quasioscillatory behavior, related to the existence of accelerator modes [59], is the exclusive property of standard mapping and so is unlike the universal quasioscillations of chaotic transport in a stochastic layer on $\log _{10} \epsilon$ considered in this work.

\section{ACKNOWLEDGMENTS}

The author gratefully acknowledges valuable comments from Professor G. Eilenberger and Professor U. Samm, and their support.

\section{APPENDIX: RESCALING PROPERTIES NEAR THE SADDLE POINTS}

According to the geometry of the separatrix maps (see Fig. 10), maps $\hat{F}_{m n}^{(p)}$ may be obtained by consecutive applications of maps $\hat{X}_{m, m \pm 1}^{(n)}$ and $\hat{Y}_{n, n \pm 1}^{(m)}$, i.e.,

$$
\begin{aligned}
& \hat{F}_{m, n}^{(1)}=\hat{Y}_{n+1, n}^{(m)} \hat{X}_{m+1, m}^{(n+1)} \hat{Y}_{n, n+1}^{(m+1)} \hat{X}_{m, m+1}^{(n)}, \\
& \hat{F}_{m, n}^{(2)}=\hat{Y}_{n-1, n}^{(m)} \hat{X}_{m+1, m}^{(n-1)} \hat{Y}_{n, n-1}^{(m+1)} \hat{X}_{m, m+1}^{(n)}, \\
& \hat{F}_{m, n}^{(3)}=\hat{Y}_{n-1, n}^{(m)} \hat{X}_{m-1, m}^{(n-1)} \hat{Y}_{n, n-1}^{(m-1)} \hat{X}_{m, m-1}^{(n)}, \\
& \hat{F}_{m, n}^{(4)}=\hat{Y}_{n+1, n}^{(m)} \hat{X}_{m-1, m}^{(n+1)} \hat{Y}_{n, n+1}^{(m-1)} \hat{X}_{m, m-1}^{(n)}
\end{aligned}
$$

for the even sum $m+n$, and

$$
\begin{aligned}
\hat{F}_{m, n}^{(1)} & =\hat{X}_{m+1, m}^{(n)} \hat{Y}_{n+1, n}^{(m+1)} \hat{X}_{m, m+1}^{(n+1)} \hat{Y}_{n, n+1}^{(m)}, \\
\hat{F}_{m, n}^{(2)} & =\hat{Y}_{m+1, m}^{(n)} \hat{Y}_{n-1, n}^{(m+1)} \hat{X}_{m, m+1}^{(n-1)} \hat{Y}_{n, n-1}^{(m)}, \\
\hat{F}_{m, n}^{(3)} & =\hat{Y}_{m-1, m}^{(n)} \hat{Y}_{n-1, n}^{(m-1)} \hat{X}_{m, m-1}^{(n-1)} \hat{Y}_{n, n-1}^{(m)}, \\
\hat{F}_{m, n}^{(4)} & =\hat{X}_{m-1, m}^{(n)} \hat{Y}_{n+1, n}^{(m-1)} \hat{X}_{m, m-1}^{(n+1)} \hat{Y}_{n, n+1}^{(m)}
\end{aligned}
$$

for the odd sum $m+n$.

We study a transformation of maps (A1) and (A2) with respect to the rescaling transformation (23). For maps the latter may be formulated as

$$
\begin{gathered}
\epsilon \rightarrow \lambda^{1 / 2} \epsilon, \quad \chi \rightarrow \chi-\pi / 2, \\
H \rightarrow-\lambda^{1 / 2} H, \quad t \rightarrow-t .
\end{gathered}
$$

The last expression in the second line in Eq. (A3) corresponds to $x \rightarrow-x, y \rightarrow-y$.

Consider, first, a transformation of the separatrix maps $\hat{X}_{m, m \pm 1}^{(n)}$ and $\hat{Y}_{n, n \pm 1}^{(m)}$ with respect to $t \rightarrow-t$ and $H \rightarrow-H$. According to Eq. (43) this transformation gives a map backward to Eq. (41) with opposite signs of $H$ and the phase $\chi$, i.e.,

$$
\hat{X}_{m, m \pm 1}^{(n)}(\chi) \rightarrow \hat{X}_{m \pm 1, m}^{(n)}(-\chi)
$$

$$
\text { for } t \rightarrow-t, \quad H \rightarrow-H \text {. }
$$

On the other hand, according to Eq. (44) it follows that products of the maps $\hat{X}_{m, m \pm 1}^{(n)}$ and $\hat{Y}_{n, n \pm 1}^{(m)}$ are transformed in the following ways:

$$
\begin{gathered}
\hat{Y}_{n, n \pm 1}^{(m \pm 1)}(-\chi) \hat{X}_{m, m \pm 1}^{(n)}(-\chi) \\
\rightarrow \hat{Y}_{n \pm 1, n}^{(m)}(-\chi) \hat{X}_{m \mp 1, m}^{(n \pm 1)}(-\chi), \\
\hat{X}_{m, m \pm 1}^{(n \pm 1)}(-\chi) \hat{Y}_{n, n \pm 1}^{(m)}(-\chi) \\
\rightarrow \hat{Y}_{m \mp 1, m}^{(n)}(-\chi) \hat{Y}_{n \mp 1, n}^{(m \pm 1)}(-\chi) \\
\text { for } \epsilon \rightarrow \lambda^{1 / 2} \epsilon, \quad \chi \rightarrow \chi-\pi / 2, \quad H \rightarrow \lambda^{1 / 2} H,
\end{gathered}
$$

which are similar to property (32) in the case of a system with a single saddle point. Therefore, the entire rescaling transformation (A3) transform the maps $\hat{F}_{m n}^{(p)}$ in the following way. Using Eqs. (39), (A1), and (A5), one can show that, for even $m+n$,

$$
\begin{aligned}
\hat{F}_{m, n}^{(1)} & =\hat{Y}_{n+1, n}^{(m)} \hat{X}_{m+1, m}^{(n+1)} \hat{Y}_{n, n+1}^{(m+1)} \hat{X}_{m, m+1}^{(n)} \\
& \rightarrow \hat{Y}_{n, n+1}^{(m)} \hat{X}_{m-1, m}^{(n)} \hat{Y}_{n+1, n}^{(m-1)} \hat{X}_{m, m-1}^{(n+1)}=\hat{F}_{m, n+1}^{(3)}, \\
\hat{F}_{m, n}^{(2)} & =\hat{Y}_{n-1, n}^{(m)} \hat{X}_{m+1, m}^{(n-1)} \hat{Y}_{n, n-1}^{(m+1)} \hat{X}_{m, m+1}^{(n)} \\
& \rightarrow \hat{Y}_{n, n+1}^{(m)} \hat{X}_{m-1, m}^{(n)} \hat{Y}_{n+1, n}^{(m-1)} \hat{X}_{m, m-1}^{(n+1)}=\hat{F}_{m, n+1}^{(4)}, \\
\hat{F}_{m, n}^{(3)}= & \hat{Y}_{n-1, n}^{(m)} \hat{X}_{m-1, m}^{(n-1)} \hat{Y}_{n, n-1}^{(m-1)} \hat{X}_{m, m-1}^{(n)} \\
\rightarrow & \hat{Y}_{n+2, n+1}^{(m)} \hat{X}_{m+1, m}^{(n+2)} \hat{Y}_{n+1, n+2}^{(m+1)} \hat{X}_{m, m+1}^{(n+1)}=\hat{F}_{m, n+1}^{(1)}, \\
\hat{F}_{m, n}^{(4)} & =\hat{Y}_{n+1, n}^{(m)} \hat{X}_{m-1, m}^{(n+1)} \hat{Y}_{n, n+1}^{(m-1)} \hat{X}_{m, m-1}^{(n)} \\
& \rightarrow \hat{Y}_{n, n+1}^{(m)} \hat{X}_{m+1, m}^{(n)} \hat{Y}_{n+1, n}^{(m+1)} \hat{X}_{m, m+1}^{(n+1)}=\hat{F}_{m, n+1}^{(2)} .
\end{aligned}
$$

From Eqs. (A7) it also follows that

$$
\begin{aligned}
& \hat{F}_{m+1, n+1}^{(1)} \rightarrow \hat{F}_{m+1, n+2}^{(3)} \equiv \hat{F}_{m+1, n}^{(3)}, \\
& \hat{F}_{m+1, n+1}^{(2)} \rightarrow \hat{F}_{m+1, n+2}^{(4)} \equiv \hat{F}_{m+1, n}^{(4)}, \\
& \hat{F}_{m+1, n+1}^{(3)} \rightarrow \hat{F}_{m+1, n+2}^{(1)} \equiv \hat{F}_{m+1, n}^{(2)}, \\
& \hat{F}_{m+1, n+1}^{(4)} \rightarrow \hat{F}_{m+1, n+2}^{(2)} \equiv \hat{F}_{m+1, n}^{(2)} .
\end{aligned}
$$

Using Eqs. (A2), similarly to Eqs. (A7), one can obtain the following transformation properties for odd $m+n$ :

$$
\begin{array}{ll}
\hat{F}_{m, n}^{(1)} \rightarrow \hat{F}_{m+1, n}^{(3)}, & \hat{F}_{m, n}^{(2)} \rightarrow \hat{F}_{m+1, n}^{(4)}, \\
\hat{F}_{m, n}^{(3)} \rightarrow \hat{F}_{m+1, n}^{(1)}, & \hat{F}_{m, n}^{(4)} \rightarrow \hat{F}_{m+1, n}^{(2)} .
\end{array}
$$

Transformation properties (A7) and (A9) of the phase space near the four saddle points with respect to the rescaling transformations (A3) are fully equivalent to the rescaling properties (23) and (24) found by the numerical integration of Hamiltonian system [Eqs. (16) and (21)]. 
[1] H. Poincaré, New Methods of Celestial Mechanics (AIP, New York, 1992).

[2] B. V. Chirikov, Phys. Rep. 52, 256 (1979).

[3] A. J. Lichtenberg and Lieberman, Regular and Stochastic Motion (Springer-Verlag, New York, 1983).

[4] J. Guckenheimer and P. Holmes, Nonlinear Oscillations, Dynamical Systems, and Bifurcations of Vector Fields (SpringerVerlag, New York, 1983).

[5] H. Aref, J. Fluid Mech. 143, 1 (1984).

[6] H. Aref, J. Fluid Mech. 173, 15 (1986).

[7] J. M. Ottino, The Kinematics of Mixing: Stretching, Chaos, and Transport (Cambridge University Press, Cambridge, 1989).

[8] T. H. Solomon and J. P. Gollub, Phys. Rev. A 38, 6280 (1988).

[9] A. L. Bertozzi, SIAM (Soc. Ind. Appl. Math.) J. Math. Anal. 19, 1271 (1988).

[10] J. B. Weiss and E. Knobloch, Phys. Rev. A 40, 2579 (1989).

[11] J. B. Weiss, Phys. Fluids A 3, 1379 (1991).

[12] D. del Castillo-Negrete and P. J. Morrison, Phys. Fluids A 5, 948 (1993).

[13] G. M. Zaslavsky, D. Stevens, and H. Weitzner, Phys. Rev. E 48, 1683 (1993).

[14] T. Ahn and S. Kim, Phys. Rev. E 49, 2900 (1994).

[15] E. Weeks, J. S. Urbach, and H. Swinney, Physica D 97, 291 (1996).

[16] D. del Castillo-Negrete, Phys. Fluids 10, 576 (1998).

[17] A. Boozer, Phys. Fluids 26, 1288 (1983).

[18] R. Cary and R. G. Littlejohn, Ann. Phys. (N.Y.) 151, 1 (1983).

[19] M. N. Rosenbluth, R. Z. Sagdeev, J. B. Taylor, and G. M. Zaslavsky, Nucl. Fusion 6, 297 (1966).

[20] A. B. Rechester and M. N. Rosenbluth, Phys. Rev. Lett. 40, 38 (1978).

[21] A. Boozer and A. B. Rechester, Phys. Fluids 21, 682 (1978).

[22] N. Pomphrey and A. Reiman, Phys. Fluids B 4, 933 (1992).

[23] R. Balescu, Phys. Rev. E 51, 4807 (1995).

[24] G. Zimbardo and P. Veltri, Phys. Rev. E 51, 1412 (1995).

[25] F. D. Angelo and R. Paccagnella, Phys. Plasmas 3, 2353 (1996).

[26] V. K. Melnikov, Trans. Moscow Math. Soc. 12, 1 (1963).

[27] N. N. Filonenko and G. M. Zaslavsky, Zh. Éksp. Teor. Fiz. 54, 1590 (1968) [Sov. Phys. JETP 27, 851 (1968)].

[28] Hamiltonian Dynamical Systems-A Reprint Selection, edited by R. S. MacKay and J. D. Meiss (Hilger, Bristol, 1987).

[29] D. F. Escande, Phys. Rep. 121, 165 (1985).

[30] C. F. F. Karney, Physica D 8, 360 (1983).

[31] B. V. Chirikov and D. L. Shepelyansky, Physica D 13, 395 (1984); B. V. Chirikov, Chaos, Solitons and Fractals 1, 79 (1991).

[32] J.-P. Bouchaud and A. Georges, Phys. Rep. 195, 127 (1990).

[33] M. F. Shlesinger, G. M. Zaslavsky, and J. Klafter, Nature
(London) 363, 31 (1993); J. Klafter, M. F. Shlesinger, and G. Zumofen, Phys. Today 49(2), 33 (1996).

[34] S. Wiggins, Chaotic Transport in Dynamical Systems (Springer-Verlag, New York, 1992).

[35] G. Zumofen and J. Klafter, Phys. Rev. E 47, 851 (1993); J. Klafter and G. Zumofen, ibid. 49, 4873 (1994).

[36] R. Balescu, Phys. Rev. E 55, 2465 (1997).

[37] S. S. Abdullaev and G. M. Zaslavsky, Bull. Am. Phys. Soc. 39, 1659 (1994); G. M. Zaslavsky and S. S. Abdullaev, Phys. Rev. E 51, 3901 (1995).

[38] S. S. Abdullaev and G. M. Zaslavsky, Phys. Plasmas 2, 4533 (1995).

[39] S. S. Abdullaev, Phys. Lett. A 234, 281 (1997).

[40] S. S. Abdullaev and K. H. Spatschek, Phys. Rev. E 60, R6287 (1999).

[41] J. Pedlosky, Geophysical Fluid Dynamics (Springer-Verlag, New York, 1982).

[42] W. H. Press, S. A. Teukolsky. W.T. Vetterling, and B. P. Flannery, Numerical Recipes, 2nd ed. (Cambridge University Press, Cambridge, 1992).

[43] D. F. Escande, in Proceedings of the International Workshop on Plasma Theory and Nonlinear and Turbulent Processes in Physics, Kiev, 1987, edited by V. G. Bar'yakhtar, V. M. Chernousenko, N. S. Erokhin, A. G. Sitenko, and A. V. Zakharov (World Scientific, Singapore, 1988), pp. 398-430.

[44] S. S. Abdullaev and G. M. Zaslavsky, Phys. Plasmas 3, 516 (1996).

[45] S. S. Abdullaev, J. Phys. A 32, 2745 (1999).

[46] V. V. Afanasiev, A. A. Chernikov, R. Z. Sagdeev, and G. M. Zaslavsky, Phys. Lett. A 144, 229 (1990).

[47] A. Lichtenberg and B. Wood, Phys. Rev. A 39, 2153 (1989).

[48] T. Geisel, A. Zacherl, and G. Radons, Phys. Rev. Lett. 59, 2503 (1987).

[49] G. M. Zaslavsky and B. A. Niyazov, Phys. Rep. 283, 73 (1997).

[50] R. Artuso, Physica D 131, 68 (1999).

[51] B. V. Chirikov, Lect. Notes Phys. 179, 29 (1983).

[52] N. W. Murray, Physica D 52, 220 (1991).

[53] B. V. Chirikov and D. L. Shepelyansky, Phys. Rev. Lett. 82, 528 (1999).

[54] D. Treshev, Physica D 116, 21 (1998).

[55] Y.-C. Lai, M. Ding, and C. Grebogi, Phys. Rev. E 47, 86 (1993).

[56] Ph. Ghendrih, A. Grosman, and H. Capes, Plasma Phys. Controlled Fusion 38, 1653 (1996).

[57] A. B. Rechester and R. B. White, Phys. Rev. Lett. 44, 1586 (1980).

[58] A. B. Rechester, M. N. Rosenbluth, and R. B. White, Phys. Rev. A 23, 2664 (1981).

[59] C. F. Karney, A. B. Rechester, and R. B. White, Physica D 4, 425 (1982). 